\title{
Fiber Optic Displacement Sensors and Their Applications
}

\author{
S. W. Harun ${ }^{1,2}$, M. Yasin ${ }^{1,3}$, H. Z. Yang ${ }^{1}$ and H. Ahmad 1 \\ ${ }_{1}^{1}$ Photonic Research Center, University of Malaya, Kuala Lumpur \\ 2Department of Electrical Engineering, Faculty of Engineering, \\ University of Malaya, Kuala Lumpur \\ ${ }^{3}$ Department of Physics, Faculty of Science and Technology, \\ Airlangga University, Surabaya \\ 1,2Malaysia \\ ${ }^{3}$ Indonesia
}

\section{Introduction}

Optical fiber-based sensor technology offers the possibility of developing a variety of physical sensors for a wide range of physical parameters (Nalwa, 2004). Compared to conventional transducers, optical fiber sensors show very high performances in their response to many physical parameters such as displacement, pressure, temperature and electric field. Recently, high precision fiber displacement sensors have received significant attention for applications ranging from industrial to medical fields that include reverse engineering and micro-assembly (Laurence et al., 1998; Shimamoto \& Tanaka, 2001); Spooncer et al., 1992; Murphy et al., 1991). This is attributed to their inherent advantages such as simplicity, small size, mobility, wide frequency capability, extremely low detection limit and non-contact properties. One of the interesting and important methods of displacement measurement is based on interferometer technique (Bergamin et al., 1993). However, this technique is quite complicated although it can provide very good sensitivity. Alternatively, an intensity modulation technique can be used in conjunction with a multimode fiber as the probe. The multimode fiber probes are preferred because of their coupling ability, large core radius and high numerical aperture, which allow the probe to receive a significant amount of the reflected or transmitted light from a target (Yasin et al., 2009; Yasin et al., 2010; Murphy et al., 1994). For future applications, there is a need for better resolution, longer range, better linearity, simple construction and low cost unit.

In this chapter, fiber-optic displacement sensors (FODS) are demonstrated using an intensity modulation technique. This technique is one of the simplest techniques for the displacement measurement, which is based on comparing the transmitted light intensity against that of the launch light to provide information on the displacement between the probe and the target. A silicon photo-diode is used to measure the transmitted and reflected light intensity. The sensor performances are investigated for various laser sources, different probes types and arrangements and different target. The theoretical analysis and the corresponding 
results on various bundled fiber based sensors are also presented in this chapter. The application of the FODSs in liquid refractive index measurement is investigated theoretically and experimentally. In the last part of this chapter, a continue monitoring the liquid level is also demonstrated by using the FODS.

\section{FODS with beam-through technique}

The intensity based sensors can be achieved by either beam-though or reflective techniques. A change in displacement of the through-beam and reflective sensors are manifested as a variation in the transmitted light and reflected light intensity, respectively. This section demonstrates a simple design for an intensity-based displacement sensor using a multimode plastic fiber in conjunction of beam-through technique. The performances of this sensor are investigated for both lateral and axial displacements. In the sensor, light is transmitted through a transmitting fiber to a receiving fiber and the received light is then measured by a silicon detector.

Fig. 1 shows a schematic diagram of the proposed sensor, which consists of two set of fiber, one set is connected to a light source and is termed as the transmitting fiber, and the other set is connected to a silicon detector and is known as the receiving fiber. In the experiment, the transmitting fiber located opposite to the receiving fiber is moved laterally and axially. The light is scattered after travelling out from the transmitting fiber and the receiving fiber collect a portion of the scattered light to transmit into the silicon detector where its intensity is measured. The intensity of the collected light is a function of axial and lateral displacement of the fiber. The light source is a He-Ne laser with a peak wavelength of $633 \mathrm{~nm}$. The light is modulated externally by chopper with a frequency of $200 \mathrm{~Hz}$, which is connected to lock-in amplifier to reduce the dc drift and interference of ambient stray light. For an axial and lateral displacement, a flexible adjusting mechanism

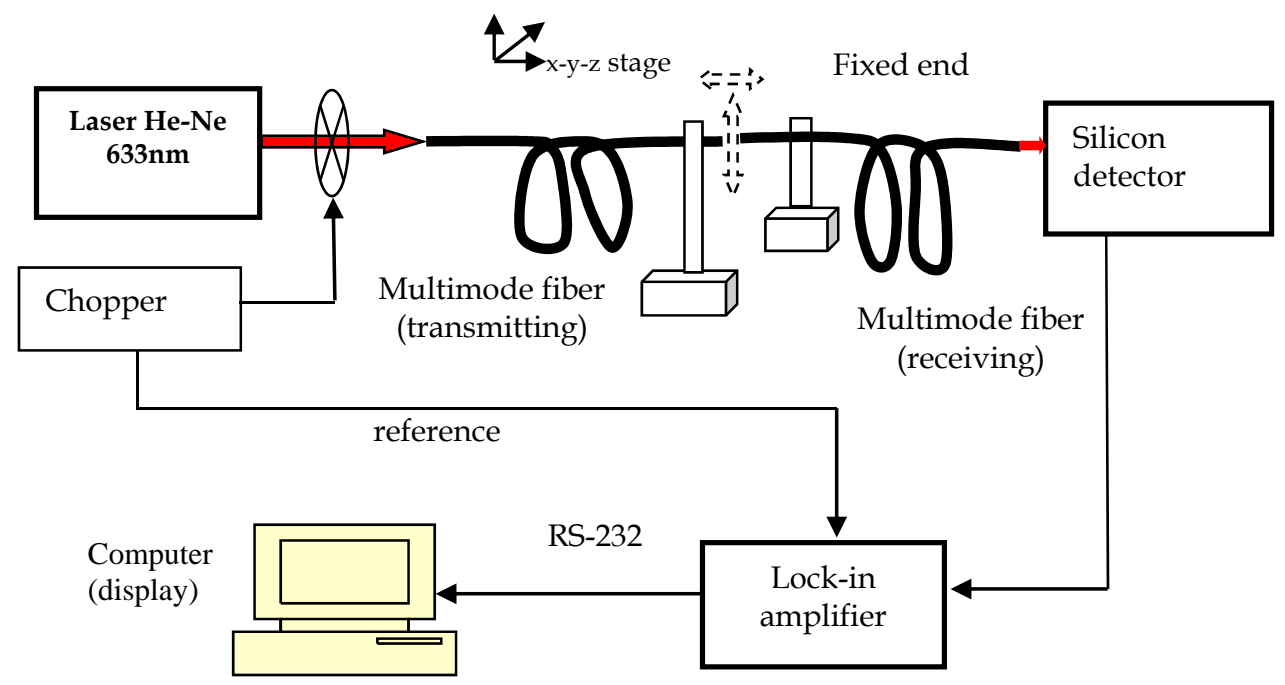

Fig. 1. Schematic diagram for lateral and axial displacement sensing using beam-through technique. 
using piezoelectric is required, so the receiving fiber tip is mounted on a translational stage, which provides fine movement of the transmitting fiber surface in the axial and lateral direction. In this experiment, the axial and lateral micro distance is varied and the lock-in amplifier output voltage of the transmitted light is directly recorded by a computer automatically using Delphi software through serial port RS232. The piezoelectric micrometer can provide precise changes of about 25 and $30 \mathrm{~nm}$ for every positive and negative pulse, respectively, and in this experiment the displacement measurement is taken in successive steps of $45 \mathrm{~nm}$.

To analyze the performance of the proposed FODS, the output voltage from a receiving fiber is related to the axial and lateral displacements of the transmitting fiber. Both fibers should be mounted perpendicular to each other and positioned flush against the surface. The output voltage of the sensor should be highest in this position. The transmitting fiber is then moved away laterally and axially from the receiving fiber tip by still maintaining perpendicularity between them. Fig. 2 shows the output voltage of the lock-in amplifier against the lateral displacement between the two ends of the fibers. In the experiment, the core diameter for both ends is varied. As expected, the voltage is highest at zero displacement from the center and the lateral movements of the transmitting fiber away from the receiving fiber resulted in a reduced output voltage as shown in the figure. The power drop pattern follows the theoretical analysis by Van Etten \& Van der Plaats (1991) in which the output transmission function is given by:

$$
\eta=\frac{2}{\pi}\left(\left(\operatorname{acrcos}\left(\frac{d}{a}\right)-\frac{d}{a} \sqrt{1-\left(\frac{d}{a}\right)^{2}}\right)\right)
$$

where $\eta, d$, and $a$ are coupling efficiency, lateral displacement, and fiber core radius, respectively. $\eta$ is defined as the ratio of output voltage over the maximum voltage. The sensitivity of the sensor is determined by a slope of a straight-line portion in the curves.

As shown in Fig. 2, the beam-through type of sensor has two symmetrical slopes and the sensitivity is higher at the smaller core diameter. At core diameter of $0.5 \mathrm{~mm}$ for both transmitting and receiving fibers, the sensitivity is obtained at around $0.0008 \mathrm{mV} / \mu \mathrm{m}$ and the slope shows a good linearity of more than $99 \%$ within a range of $420 \mu \mathrm{m}$. The linear range increases to around $800 \mu \mathrm{m}$ for the both slopes as the core diameter increased to 1.0 $\mathrm{mm}$. The linear range can be further increased to more than $1000 \mu \mathrm{m}$ by using a larger core for the receiving fiber as shown in Fig. 2. However, the voltage is unchanged at a small lateral displacement for this sensor due to the larger receiving core, which covers the whole diverged beam from the transmitting fiber. The highest resolution of $13 \mu \mathrm{m}$ is obtained with core diameter of $0.5 \mathrm{~mm}$ for both fibers. In this work, the resolution is defined as the minimum displacement which can be detected by this sensor.

The performance of the sensor with lateral displacement is summarized as shown in Table 1. The output voltage from a receiving fiber is related to axial displacement of end surface of the transmitting fiber. Fig. 3 shows the output voltage of the lock-in amplifier against the axial displacement for the different core diameters. In this experiment, the end surface of transmitting fiber is moved away from the receiving fiber tip by still maintaining perpendicularity between them. As expected, the voltage is highest at zero displacement from the center and the output voltage reduces as the axial displacement increases for all 


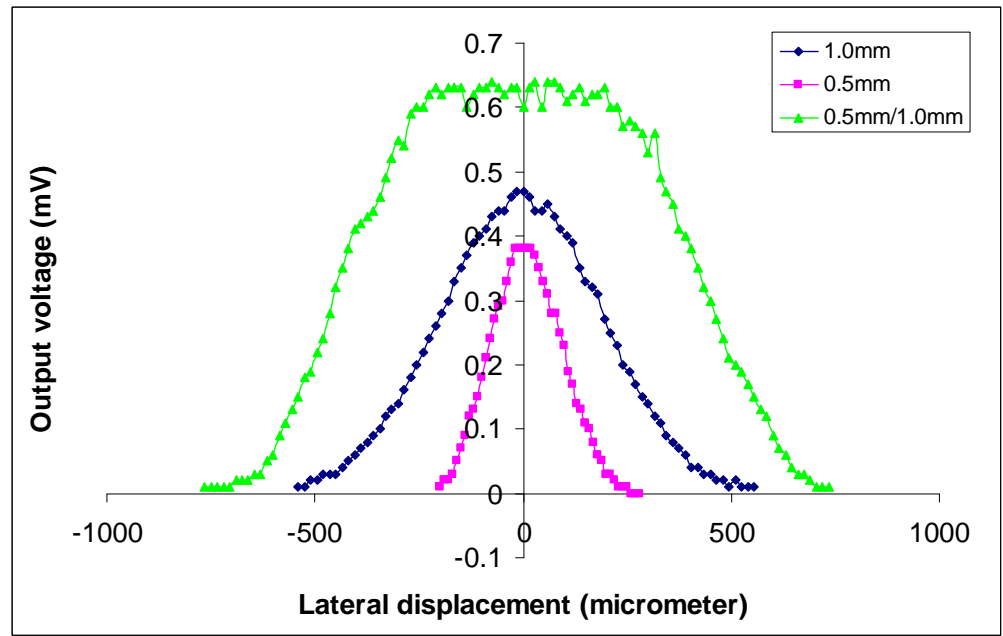

Fig. 2. The output voltage of the lock-in amplifier against the lateral displacement of the transmitting fiber.

\begin{tabular}{|c|c|c|c|c|c|}
\hline \multirow[b]{2}{*}{$\begin{array}{c}\text { Fiber's core } \\
\text { diameter }\end{array}$} & \multicolumn{2}{|c|}{ The left slope } & \multicolumn{2}{|c|}{ The right slope } & \multirow[b]{2}{*}{$\begin{array}{l}\text { Resolution } \\
\quad(\mu \mathrm{m})\end{array}$} \\
\hline & $\begin{array}{r}\text { Sensitivity } \\
(\mathrm{mV} / \mu \mathrm{m})\end{array}$ & $\begin{array}{c}\text { Linear range } \\
(\mu \mathrm{m})\end{array}$ & $\begin{array}{l}\text { Sensitivity } \\
(\mathrm{mV} / \mu \mathrm{m})\end{array}$ & $\begin{array}{c}\text { Linear range } \\
(\mu \mathrm{m})\end{array}$ & \\
\hline $\begin{array}{l}1.0 \mathrm{~mm} \\
/ 1.0 \mathrm{~mm}\end{array}$ & 0.0003 & $855(585-1440)$ & 0.0004 & $\begin{array}{c}765 \text { (1845- } \\
2610)\end{array}$ & 33 \\
\hline $\begin{array}{l}0.5 \mathrm{~mm} \\
/ 0.5 \mathrm{~mm} \\
\end{array}$ & 0.0008 & $420(120-540)$ & 0.0007 & $\begin{array}{c}420(750- \\
1170) \\
\end{array}$ & 13 \\
\hline $\begin{array}{l}0.5 \mathrm{~mm} \\
/ 1.0 \mathrm{~mm}\end{array}$ & 0.0005 & $1035(450-1485)$ & 0.0005 & $\begin{array}{c}1125(3060- \\
4185)\end{array}$ & 20 \\
\hline
\end{tabular}

Table 1. Performance of the Lateral Displacement Sensor

core diameters used. The power drop pattern follows the theoretical analysis from (Van Etten \& Van der Plaats, 1991) in which the output transmission function is given by:

$$
\eta \approx 1-\frac{z}{a} \frac{2}{\pi(N A)^{2}}\left(\arcsin (N A)-N A \sqrt{1-(N A)^{2}}\right)
$$

where $\eta, z, a$, and NA are coupling efficiency, axial displacement, core radius, and numerical aperture, respectively.

As shown in Fig. 3, the sensors only have one slope and the sensitivity is higher at the smaller core diameter. At core diameter of $0.5 \mathrm{~mm}$ (for both transmitting and receiving fibers), the sensitivity is obtained at around $0.0002 \mathrm{mV} / \mu \mathrm{m}$, which is the highest and the slope shows a good linearity of more than $99 \%$ within a range of $900 \mu \mathrm{m}$. The linear range increases to $3195 \mathrm{~m}$ with the larger core diameter of $1.0 \mathrm{~mm}$. In case of the receiving core is bigger than the transmitting core, the voltage is almost constant at small axial displacements due to the coherent light source, which has a small divergence angle. The highest resolution 


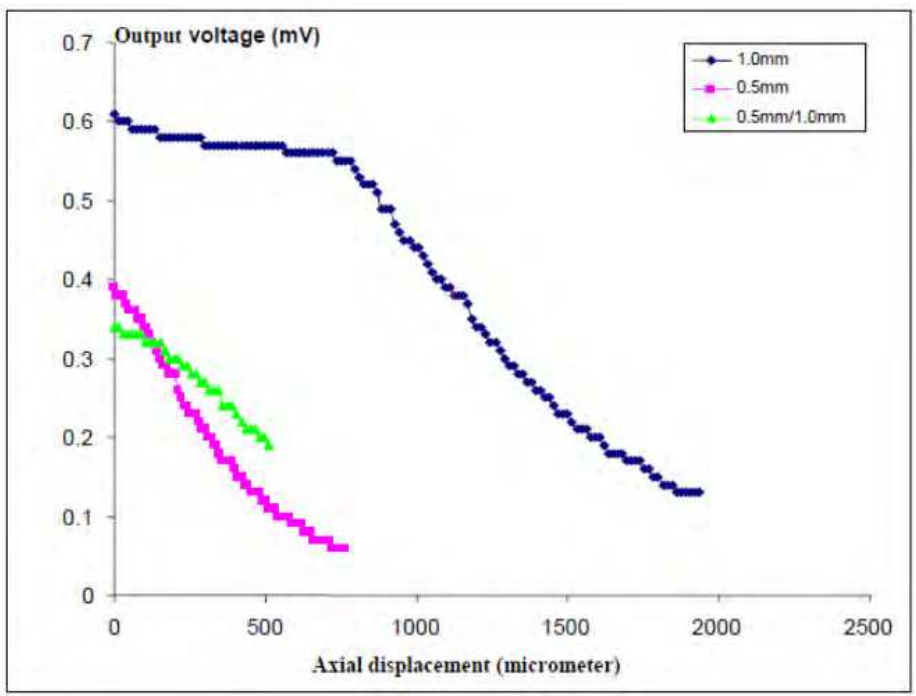

Fig. 3. The output voltage of the lock-in amplifier against the axial displacement of the transmitting fiber.

is obtained at $50 \mathrm{~m}$ with $0.5 \mathrm{~mm}$ core diameter for both fibers. The performance of the sensors with axial displacement is summarized as shown in Table 2. The stability of these sensors is observed to be less than $0.01 \mathrm{mV}(3 \%)$. The experimental results are capable of offering quantitative guidance for the design and implementation of the displacement sensor. This sensor requires two probes, which is precisely aligned and therefore the applications are limited. In the next section, various reflective types of FODSs are proposed to improve the sensitivity, linearity and dynamic range of the sensor.

\begin{tabular}{|c|c|c|c|}
\hline Fiber's core diameter & Sensitivity $(\mathrm{mV} / \mu \mathrm{m})$ & Linear range $(\mu \mathrm{m})$ & Resolution $(\mu \mathrm{m})$ \\
\hline \hline $1.0 \mathrm{~mm} / 1.0 \mathrm{~mm}$ & 0.0001 & $3195(2340-5535)$ & 100 \\
\hline $0.5 \mathrm{~mm} / 0.5 \mathrm{~mm}$ & 0.0002 & $900(0-900)$ & 50 \\
\hline $0.5 \mathrm{~mm} / 1.0 \mathrm{~mm}$ & 0.0001 & $1530(0-1530)$ & 100 \\
\hline
\end{tabular}

Table 2. Performance of the Axial Displacement Sensor

\section{FODS with reflective configuration}

The FODS based on intensity modulation and reflective arrangement provides a promising solution for displacement measurement in terms of wide dynamic range, with high potential for ultra-precise non-contact sensing. It also provides flexibility in incorporating the optical sensors permanently into composite structures for monitoring purpose [Wang et al., 1997). In the simplest design of reflective FODS, a probe with a pair of fibers is normally used as the media to transfer/collect the light to/from the target and its theoretical analysis is fully contributed (Faria, 1998). In the design of FODS system the sensor probe is playing a majority role comparison with the selection of laser source and reflector. Hence, the researchers are paid more attentions in the development of sensor probe to improve the 
performance of FODS. The FODSs described in this section are focus on the various configurations of sensor probe since they are mainly influence the performance of FODS.

\subsection{FODS using a probe with two receiving fibers}

In this section, a new configuration of the FODS is reported by using two receiving fibers which are bundled together. The mathematical analysis of FODS is developed to simulate the theoretical results, which is then compared to the experimental result. The performance of this FODS is also compared experimentally with the conventional FODS. The probe structure of the proposed FODS is shown in Fig. 4. It consists of one transmitting and two receiving plastic multi-mode fibers bundled together in parallel. To analyze the theory of this sensor setup, a more realistic approach - Gaussian beam is used to describe the light leaving the transmitting fiber. The irradiance of emitted light is obeying an exponential law according to

$$
I(r, z)=\frac{2 P_{E}}{\pi \omega^{2}(z)} \exp \left(-\frac{2 r^{2}}{\omega^{2}(z)}\right)
$$

where $P_{E}$ is the emitted power from the light source, $r$ is the radial coordinate and $z$ is the longitudinal coordinate. $\omega(z)$ is the beam radius which is also a function of $z, \omega(z)=$ $\omega_{0} \sqrt{1+\left(\frac{z}{z_{R}}\right)^{2}}$. The waist radius $\omega_{0}$ and Rayleigh range $z_{R}$ are the important parameters in the Gaussian Beam function.

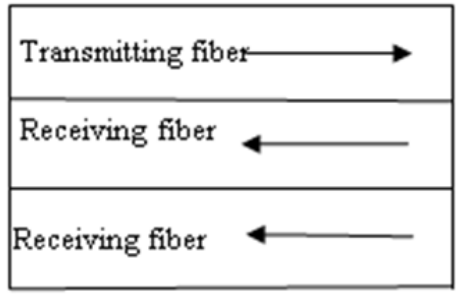

(Side View)

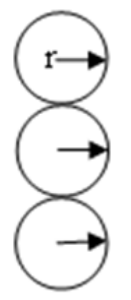

(Front View)

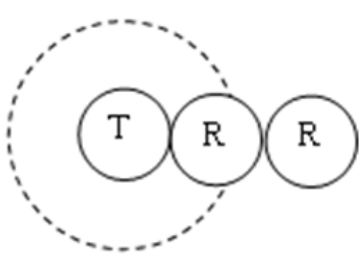

(Overlap Area View)

Fig. 4. Side, Front and Overlap Views of FODS probe structure with two receiving fibers

The optical power received by the receiving fiber can be evaluated by integrating the irradiance, $I$ over the surface area of the receiving fiber end, $S_{r}$.

$$
P(z)=\int_{S_{r}} I(r, z) d S_{r}
$$

The overlapping area of the reflected light area and the core of the receiving fibers is also illustrated in Fig 4 . The power value of reflected light collected by two receiving fibers increased with the increased of displacement of probe and target mirror. Two receiving fibers collected the reflecting light results the receiving light power increased. Based on this geometrical analysis two receiving fibers collected the reflected light significantly affects the transfer function of the FODS. 
The Eq. (3) can be described in other expressions in order to simulate conveniently. The power collected by the first and second receiving fibers are denoted as $P_{1}$ and $P_{2}$, respectively where $P_{1}$ is closer to the transmitting fiber. From Eq. (3), $P_{1}$ and $P_{2}$ can also be written as;

$$
\begin{gathered}
P_{1}(z)=\frac{2 P_{E}}{\pi \omega^{2}(z)} \int_{y=-R_{r}}^{R_{r}} \int_{x=R_{t}+R_{r}-\sqrt{R_{r}^{2}-y^{2}}}^{R_{t}+R_{r}+\sqrt{R_{r}^{2}-y^{2}}} \exp \left(-\frac{2\left(x^{2}+y^{2}\right)}{\omega^{2}(z)}\right) d x d y \\
P_{1}(z)=\frac{2 P_{E}}{\pi \omega^{2}(z)} \int_{y=-R_{r}}^{R_{r}} \int_{x=R_{d}+R_{t}+R_{r}-\sqrt{R_{r}^{2}-y^{2}}}^{R_{d}+R_{t}+R_{r}+\sqrt{R_{r}^{2}-y^{2}}} \exp \left(-\frac{2\left(x^{2}+y^{2}\right)}{\omega^{2}(z)}\right) d x d y
\end{gathered}
$$

where the $R_{t}, R_{r}$ is the radius of transmitting fiber and receiving fiber, respectively. The $R_{d}$ is the diameter of the receiving fiber. The radial coordinate $r$ is expressed by $\sqrt{x^{2}+y^{2}}$ in Cartesian coordinate system. Then the total amount of power collected by both receiving fibers is;

$$
P=P_{1}+P_{2}
$$

The conventional FODS only collects power $P_{1}$ using a pair of fibers bundled together. Compared to this sensor, the proposed FODS collects more reflected light due the additional $P_{2}$ power, which increases the dynamic range of the sensor.

The software simulation is programmed and implemented in MATLAB. Some important parameters are specified in the programming, wavelength of the laser source $\lambda=594 \mathrm{~nm}$, transmitting fiber core radius $R_{t}=0.5 \mathrm{~mm}$ and numerical aperture value $\mathrm{NA}=0.25$. Fiber diameter $R_{d}=2 \mathrm{~mm}$. The theoretical analysis transfer function of proposed displacement sensor in Eq. (6) can be normalized by its maximum collected power $P_{\max }, P_{\mathrm{n}}=P / P_{\max }$. The normalized distance $h_{n}=h / z$, while power increase to maximum for small values $h$ from 0 to $z$, power decrease to zero according to the $\mathrm{h}$ values higher than $\mathrm{z}$. The simulation results are then compared with the experimental results.

The experiment setup for the FODS with two receiving fibers is shown in Fig. 5. It consists of a light source, a chopper, a sensor probe, a flat mirror, a silicon detector, a lock-in amplifier and a PC. The sensor probe consists of one transmitting and two receiving plastic multimode fibers which are bundled together in parallel. The transmitting and receiving fiber length is $2 \mathrm{~m}$ with a core diameter of $1 \mathrm{~mm}$. A $594 \mathrm{~nm}$ yellow He-Ne laser is used as a light source. It has the maximum output power of $4 \mathrm{~mW}$ and beam divergence of $0.92 \mathrm{mRads}$, which is modulated by external chopper with a frequency $200 \mathrm{~Hz}$. The transmitting end of fiber probe radiates the modulated light from the laser to the mirror. The flat mirror is controlled by a piezoelectric motor and driver. The distance between the fiber probe and the mirror is varied in successive steps of $4 \mu \mathrm{m}$ and the light voltage which is represented the optical power is measured against the change in the mirror displacement stage. Then the mirror reflects the transmitting light into the receiving end of fiber probe. The reflected light through receiving end can be detected by the silicon detector. The photon energy collected by detector is converted into a voltage. The output of the detector transfers into the lock-in amplifier to deduce the dc drift and filter out the undesired noise. The lock-in amplifier is connected to a PC using RS232 data series line. From the PC, the output light voltage is monitored. 


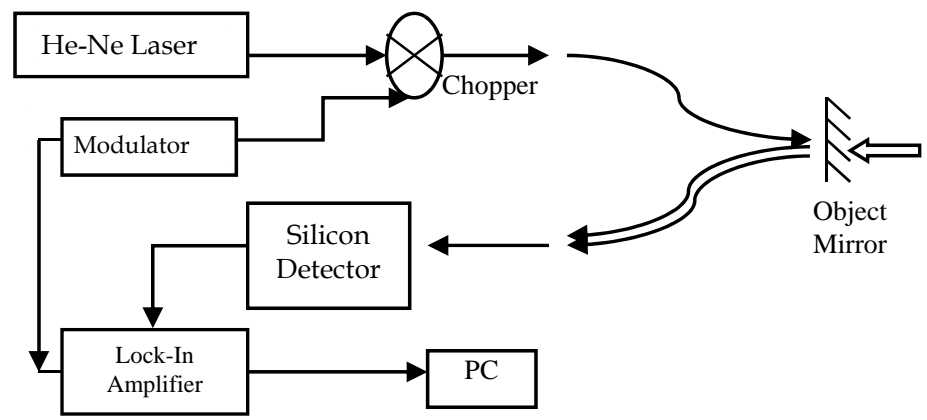

Fig. 5. Experiment setup of proposed FODS with two receiving fibers.

In both theoretical and experimental analysis, the results are processed and displayed in the normalized forms which the output power is normalized by the maximum collected optical power and the displacement is normalized by the distance parameter $z$. This is to make the output function a dimensionless function and eliminate the dependency of the FODS output function to the fiber core radius and divergence angle. Fig. 6 shows the comparison of the simulated and the observed response of the proposed FODS. As shown in Fig. 6, both curves exhibit a maximum with a steep linear front slope and back slope which follows an almost inverse square law relationship for the reflected light intensity versus distance of the mirror from transmitting fiber end. The signal is minimal at zero distance because the light cone does not reach the core of both receiving fibers. As the displacement increases, the size of cone of the reflected light at the plane of fiber also increases, which then starts to overlap with the receiving fiber cores leading to a small output voltage. Further increases in the displacement lead to larger overlapping which in turn results in an increase in the output voltage. However, after reaching the maximum value, the output voltage starts decreasing even though the displacement increases. This is due to the large increase in the size of the light cone and the power density decreases with the increase in the size of the cone of light.

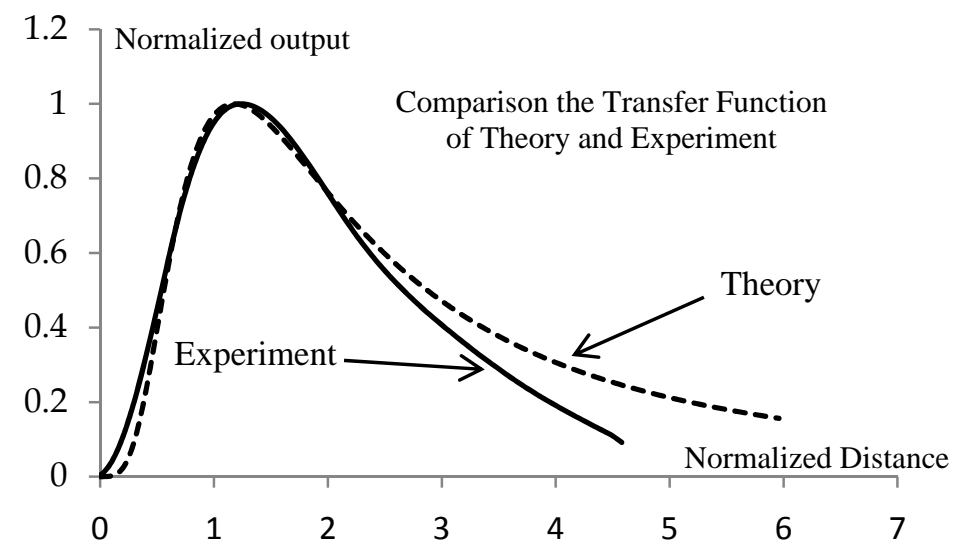

Fig. 6. Normalized collected optical power versus the normalized displacement curves for both theoretical and experimental results. 
The maximum normalized output powers of 1 are obtained at the normalized displacement distances of 1.2 for the theoretical curve and 1.3 for the experimental curve as shown in Fig. 6. The close agreement of theoretical and experimental results in the Fig. 6 is quite evident. The small difference can be accounted for experimental limitations mainly due to the geometry error of the fiber used and positioning error. Table 3 summarizes the performance comparison of the simulated and the experimental result of the sensor. The slope of the response curve is the sensitivity, which is expressed in the unit of $\mathrm{mW} / \mu \mathrm{m}$. As shown in the table, the linearity range at the back slope is nearly 3 times that of the linearity range at the front slope. However, the sensitivity at the front slope is nearly $3 \sim 4$ times higher than that of the sensitivity at the back slope.

\begin{tabular}{|c|c|c|c|c|}
\hline \multirow{2}{*}{ Methods } & \multicolumn{2}{|c|}{ The front slopes } & \multicolumn{2}{c|}{ The back slopes } \\
\cline { 2 - 5 } & Linearity range & Sensitivity & $\begin{array}{c}\text { Linearity } \\
\text { range }\end{array}$ & Sensitivity \\
\hline \hline Theoretical & $\begin{array}{c}0.8 \mu \mathrm{m} \\
(0.3 \sim 1.1)\end{array}$ & $0.06 \mathrm{mV} / \mu \mathrm{m}$ & $\begin{array}{c}2.5 \mu \mathrm{m} \\
(1.4 \sim 3.9)\end{array}$ & $\begin{array}{c}0.015 \mathrm{mV} / \mu \\
\mathrm{m}\end{array}$ \\
\hline Experimental & $\begin{array}{c}0.9 \mu \mathrm{m} \\
(0.2 \sim 1.1)\end{array}$ & $0.06 \mathrm{mV} / \mu \mathrm{m}$ & $\begin{array}{c}2.5 \mu \mathrm{m} \\
(1.5 \sim 4.0)\end{array}$ & $\begin{array}{c}0.017 \mathrm{mV} / \mu \\
\mathrm{m}\end{array}$ \\
\hline
\end{tabular}

Table 3 . The performance comparison between theoretical and experiment results.

The experiment is also repeated for the conventional sensor with one transmitting and one receiving fibers. The response of the conventional FODS is then compared to that of the proposed FODS as shown in Fig. 7. As shown in the figure, the maximum power collected by the receiver is obtained at a shorter distance for the conventional sensor. The sensor performance comparison between the proposed and the conventional sensors is summarized in Table 4 . The sensitivity of both sensors is almost similar. However, the proposed sensor obviously has a better linearity range as shown in Table 4. This is attributed to the amount of the collected light intensity, which is higher in the proposed sensor compared to that of the conventional one.

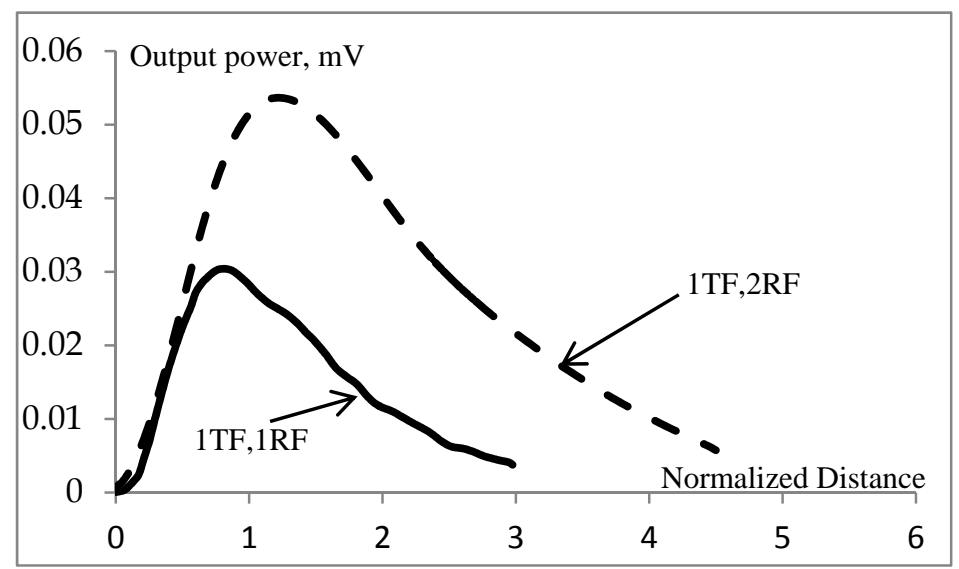

Fig. 7. The displacement curves for both proposed (1TF, 2RF) and conventional (1TF, 1RF) sensors 


\begin{tabular}{|c|c|c|c|c|}
\hline \multirow{2}{*}{ Methods } & \multicolumn{2}{|c|}{ The front slopes } & \multicolumn{2}{c|}{ The back slopes } \\
\cline { 2 - 5 } & Linearity range & Sensitivity & Linearity range & Sensitivity \\
\hline \hline Conventional & $\begin{array}{c}0.5 \mu \mathrm{m} \\
(0.2 \sim 0.7)\end{array}$ & $0.06 \mathrm{mV} / \mu \mathrm{m}$ & $\begin{array}{c}1.4 \mu \mathrm{m} \\
(0.9 \sim 2.3)\end{array}$ & $\begin{array}{c}0.016 \\
\mathrm{mV} / \mu \mathrm{m}\end{array}$ \\
\hline Proposed & $\begin{array}{c}0.9 \mu \mathrm{m} \\
(0.2 \sim 1.1)\end{array}$ & $0.06 \mathrm{mV} / \mu \mathrm{m}$ & $\begin{array}{c}2.5 \mu \mathrm{m} \\
(1.5 \sim 4.0)\end{array}$ & $\begin{array}{c}0.017 \\
\mathrm{mV} / \mu \mathrm{m}\end{array}$ \\
\hline
\end{tabular}

Table 4 . The performance comparison between the proposed and conventional sensors

The maximum linearity ranges of $2.5 \mu \mathrm{m}$ and $0.9 \mu \mathrm{m}$ are obtained at the back and front slopes respectively for the proposed sensor as shown in Table 4 . The linearity range of the proposed sensor is improved by about $44 \%$ for both slopes compared to the conventional sensor. As indicated by the above results, we can conclude that the employment of two receiving fibers increases the linearity range of the sensor, which is very useful for the large displacement measurements. Both theoretical and experimental results are capable of offering quantitative guidance for the design and implementation of the displacement sensor.

\subsection{The FODS with two asymmetrical fibers bundled}

As discussed in most of the FODSs [Ko et al., 1995; Elasar et al., 2002; Oiwa \& Nishitani, 2004; Cao et al., 2007), the radius of the transmitting and receiving fibers are often made the same for the convenience of analytical study and experiments. However, there is a lack of research work on the displacement sensor using bundled fiber with different core radius. In this work, a mathematical model of displacement sensor using asymmetrical bundled fiber is developed. Some simulations were carried out based on the mathematical model and experimental results were also obtained to validate the MATLAB simulated results. The effect of different core radial ratios (CRRs) to the dynamic range, sensitivity and illumination area of bundled fiber are analyzed and discussed in this section.

\section{Theoretical analysis}

The proposed FODS consists of a transmitting and receiving fibers as well as a reflecting mirror. Both fibers are of different core radius and are bundled together in parallels as shown in Fig. 8. Let $r_{T}$ and $r_{R}$ denote the core radius of the transmitting fiber and the core radius of the receiving fiber. The core radial ratio, CRR is the ratio of transmitting fiber and receiving fiber core radius, as given below:

$$
\mathrm{CRR}, k=\frac{r_{R}}{r_{T}}
$$
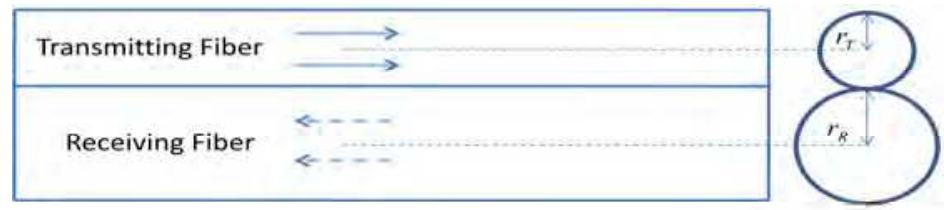

(Cross Section)

Fig. 8. Side and front views of the transmitting and receiving fiber ends. 
Fig. 9 shows the geometrically illustration of the overlapping area between the reflected light circle and the core of the receiving fiber at different CRR. Based on this figure, in the same core radius of transmitting fiber; the reflected light power collected by the receiving fiber increases with the increasing core radius of the receiving fiber. The larger receiving fiber core radius and core area, the bigger fraction of reflected light can be collected by the receiving fiber. In the previous report (Faria, 1998), two major approaches have been introduced for theoretical analysis, namely geometrical and Gaussian Beam approaches. For the former approach, the simple assumption is made that the light intensity is constant within the reflected light circle. On the other hand, the light intensity outside the reflected light circle is null. This approach is apparently less accurate compared to the second approach. Gaussian Beam approach is a more realistic and more accurate method. The intensity of the light emitted from the transmitting fiber is described with Gaussian distribution as shown in Eq. (3).
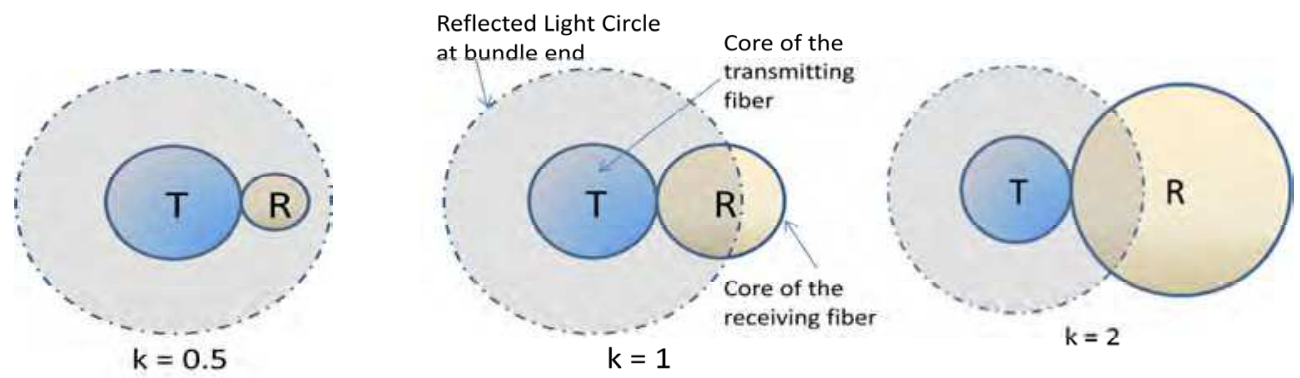

Fig. 9. Geometrical illustration for the overlapping area between the reflected light circle and the core of the receiving fiber at different CRRs.

The light power collected by the receiving fiber can be evaluated by using integral as shown in Eq. 4. However, the exact integration is tedious and impossible. Therefore, assumptions and approximation were used to dissolve the integration. For points situated in the far-field, $\mathrm{z}>>\mathrm{z}_{\mathrm{R}}$ the following relations with the divergence angle can be obtained

$$
\theta_{a}=\tan \theta_{a}=\frac{\omega(z)}{z}=\frac{\omega_{0}}{z_{R}}=\frac{\lambda}{\pi \omega_{0}}
$$

Core radius of the transmitting fiber and receiving fibers are given by the approximation

$$
\omega_{T}=z_{a} \tan \theta_{a} \approx z_{a} \theta_{a}
$$

and

$$
\omega_{R}=k \omega_{T}=k z_{a} \theta_{a}
$$

where $z_{a}$ is the distance between the beam source to the fiber end (Wang et al., 1997). The core area of the receiving fiber is computed from

$$
S_{a}=\pi \omega_{R}^{2}=\pi k^{2} z_{a}^{2} \theta_{a}^{2}
$$


The radial distance between the two core centers of the transmitting fiber and receiving fiber is determined from

$$
r=\omega_{T}+\omega_{R}=\omega_{T}+k \omega_{T}=(1+k) z_{a} \theta_{a}
$$

The path of the beam from the beam source to the bundle end after the reflection is given by

$$
z_{a}+2 h
$$

The displacement parameter in the normalized form is presented as

$$
\zeta=\frac{z_{a}+2 h}{z_{a}}
$$

or

$$
\zeta=1+2 h_{N}
$$

where $h_{N}=h / z_{a}$. To relate the displacement between the reflective mirror to the fiber end, $h$ to the transfer function, with the help of the results determined above, the collected power of the receiving fiber can be expressed as

$$
\begin{aligned}
P(h) & =\frac{2 P_{E}}{\pi \omega^{2}\left(z_{a}+2 h\right)} \exp \left(-\frac{2 r^{2}}{\omega^{2}\left(z_{a}+2 h\right)}\right) \times S_{a} \\
& =\frac{2 P_{E} k^{2} z_{a}^{2}}{\left(z_{a}+2 h\right)^{2}} \exp \left(-\frac{2\left((1+k) z_{a}\right)^{2}}{\left(z_{a}+2 h\right)^{2}}\right)
\end{aligned}
$$

By substituting Eq. (15) into this equation, we obtain

$$
P(\xi)=\frac{2 P_{E} k^{2}}{\xi^{2}} \exp \left(-\frac{2(1+k)^{2}}{\xi^{2}}\right)
$$

The maximum received power is achieved when $P^{\prime}(\xi)=0$, and this leads to

$$
\xi_{\max }=\sqrt{2}(1+k)
$$

Based on the relation in Eq. (3-16), the maximum $h$ is given by

$$
h_{\max }=\frac{\sqrt{2} k+\sqrt{2}-1}{2}
$$

The maximum power is given by

$$
P_{\max }=P(\sqrt{2}(1+k))=\frac{k^{2} P_{E}}{(k+1)^{2}} \exp (-1)
$$

In the normalized form, Eq. (3-15) is rewritten as 


$$
P_{N}(\xi)=\frac{P(\xi)}{P_{\max }}=\frac{2(k+1)^{2}}{\xi^{2}} \exp \left(1-\frac{2(1+k)^{2}}{\xi^{2}}\right)
$$

In the analysis, the theoretical model of the FODS is modeled based on the similar parameters used in the experiment: Wavelength of the laser source $\lambda=594 \mathrm{~nm}$, transmitting fiber core radius $r_{R}=0.5 \mathrm{~mm}$ and numerical aperture value $\mathrm{NA}=0.4$. Based on the same parameters, four analytical model were simulated for $\mathrm{k}=0.5,1,2$ and 3 which were based on the available fiber core radius combinations in the experiments.

\section{Experiment}

The experiment for the FODS with two asymmetrical bundled fibers is carried using the similar set-up as shown in Fig. 5, but using bundled fiber with different core radius. The asymmetrical bundled fiber is constructed by pairing two different plastic fibers with the core radiuses of either $0.25 \mathrm{~mm}$ or $0.50 \mathrm{~mm}$ or $0.75 \mathrm{~mm}$. Due to the limited selections of core diameters, six combinations were selected for the experiments: $\left[k, r_{T}, r_{R}\right]=[0.5,0.5 \mathrm{~mm}$, $0.25 \mathrm{~mm}],[1.0,0.5 \mathrm{~mm}, 0.5 \mathrm{~mm}],[2.0,0.25 \mathrm{~mm}, 0.5 \mathrm{~mm}]$ and $[3.0,0.25 \mathrm{~mm}, 0.75 \mathrm{~mm}] . k$ is the core radial ratio. A precise displacement reference between the bundle end and the reflecting mirror is imperative for the experiment. Therefore, a New Focus 9061 motorized stages which is driven by a picomotor is used to change the displacement of the reflecting mirror from the fiber probe. Each increment step in the displacement is made identical and accurate. The collected light power in the receiving fiber is converted by a silicon detector into electrical power. Lastly, the electrical signal is filtered by a lock-in amplifier and recorded in the computer.

\section{Characteristic of the sensor}

In both theoretical and experimental analysis, the results are processed and displayed in the normalized forms which the output power is normalized by the maximum output power and the displacement is normalized by the parameter $z_{a}$. This is to make the output function a dimensionless function and eliminate the dependency of the FODS output function to the fiber core radius and divergence angle. Figs. 10 and 11 show the analytical and experimental results respectively for the proposed FODS. As shown in both figures, the location of the maximum output is shifted toward the right along the axis of displacement as the value of $\mathrm{k}$ increases. Besides, the linear range on the front slope and back slope gets larger for every larger value of $k$. Both graphs exhibit the almost the same trend of characteristics in the curves as the value of $\mathrm{k}$ becomes larger. This phenomenon can be explained by the use of distinctive core radius of the two fibers. As shown in Fig. 9, at the same displacement the fraction of overlap area in receiving fiber core by the reflected light circle (percentage of shaded area in the receiving fiber core) is differ for different CRR. For a larger value of $k$, the fiber displacement sensor requires further displacement to achieve maximum overlap area. Adversely, the sensitivity of the fiber displacement sensor decreases as the CRR increases. On the other hand, some error in the initial displacement $(0<h<0.3)$ is observed if the two overlaid graphs are compared. This error is accounted to the approximation used in the theoretical analysis.

The performance of the proposed FODS from the experimental results is summarized in Table 5. The results show that the magnitude of the sensitivity decreases as the CRR or $k$ value becomes larger while the linear range is larger for a larger value of $k$. The sensitivity 


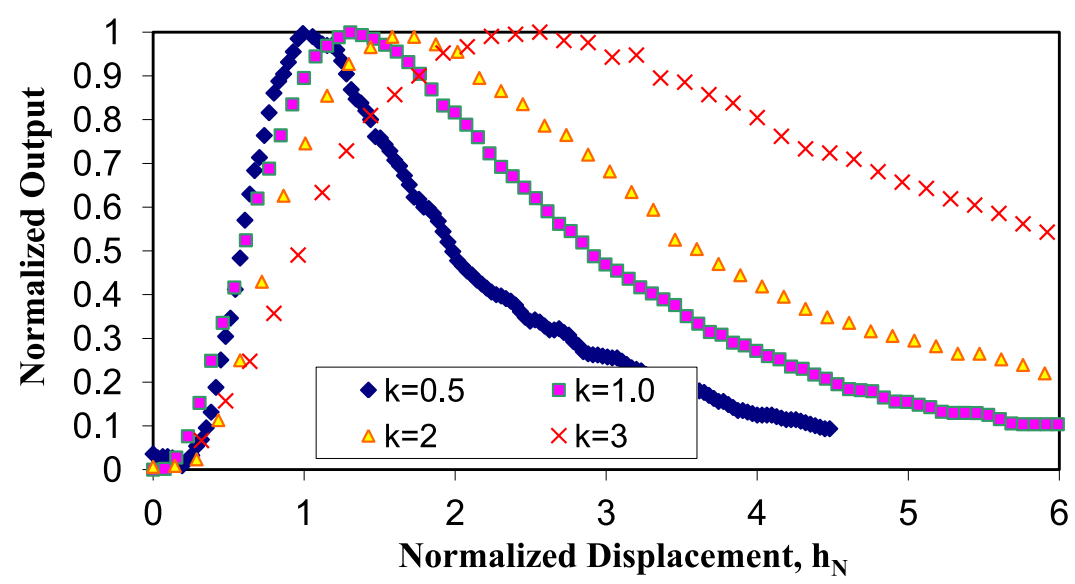

Fig. 10. The experimental result of proposed FODS model at different CRRs or k values.

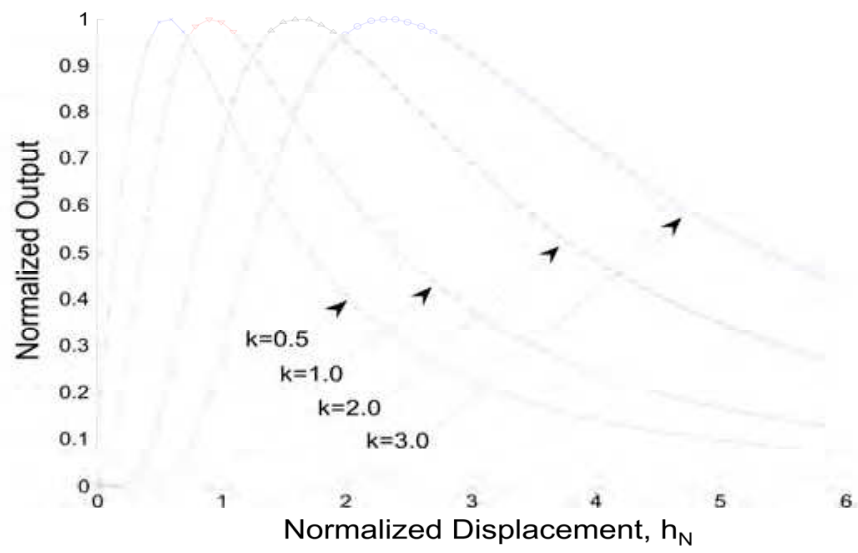

Fig. 11. The theoretical result of the fiber displacement sensor at different CRRs or $\mathrm{k}$ values.

\begin{tabular}{|c|c|c|c|c|}
\hline CRR,$k$ & $\begin{array}{c}\text { Front Slope } \\
\text { Sensitivity }\end{array}$ & $\begin{array}{c}\text { Linear Range } \\
(\mathrm{mm})\end{array}$ & $\begin{array}{c}\text { Back Slope } \\
\text { Sensitivity }\end{array}$ & $\begin{array}{c}\text { Linear Range } \\
(\mathrm{mm})\end{array}$ \\
\hline \hline 0.5 & 1.7615 & $0.320-0.800$ & -0.5270 & $1.312-1.176$ \\
\hline 1 & 1.0984 & $0.230-0.998$ & -0.3378 & $1.536-3.302$ \\
\hline 2 & 0.9688 & $0.288-1.296$ & -0.2581 & $1.728-4.320$ \\
\hline 3 & 0.6955 & $0.320-1.440$ & -0.1366 & $2.240-5.400$ \\
\hline
\end{tabular}

Table 5. The sensitivity and linear range for different $k$ values

characteristic trend is consistent with the theoretical plot as shown in Fig. 12. Fig. 12 shows the normalized sensitivity against normalized displacement at various $k$ values. The curve width of the graph represents the linear range of the sensor. As shown in the figure, the linear range of the sensor increases with the value of $k$ which is in agreement with the 


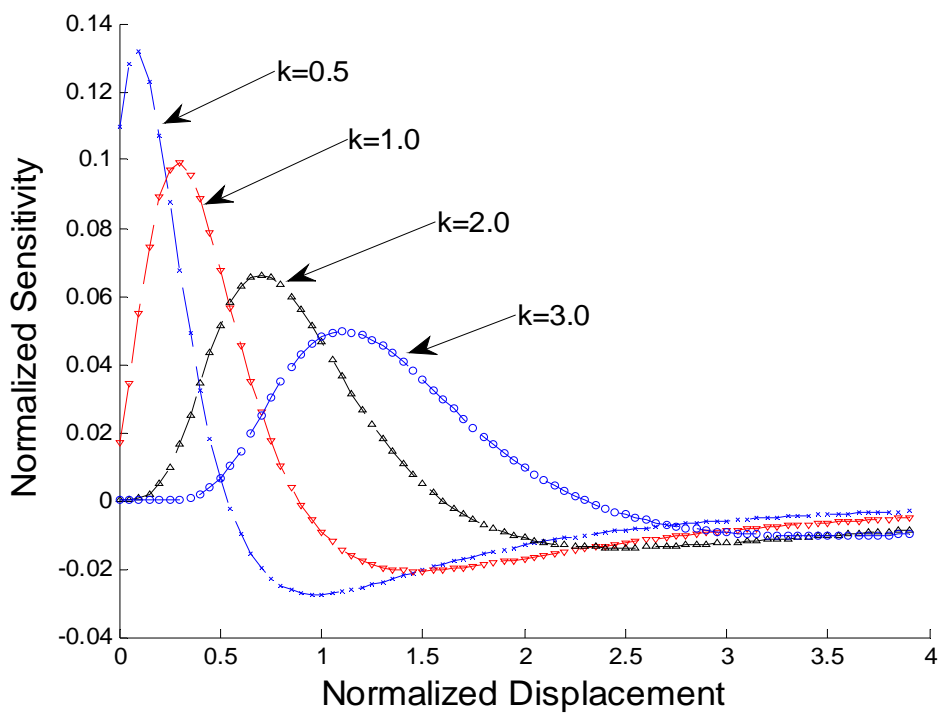

Fig. 12. Theoretical normalized sensitivity curves of the FODS at various $k$ values.

experimental result in Table 5. This property provides a greater enhancement in FODS applications in terms of flexibility, wider dynamic range and high precision displacement measurement. The maximum sensitivity of 1.76 is obtained at $k=0.5$. The largest dynamic range of Fig. 10 is obtained at $k=3$. The conventional FODS which uses two fibers with identical core radius often encounters several restrictions due to limited linear range for the measurement. Besides, the limited option of sensitivity often becomes a challenge in the high-precision measurement. This restriction can be avoided using a suitable CRR or $k$ value. The $k$ value can be chosen in a way to provide the optimum performance.

\subsection{The FODSs with two asymmetrical fibers inclined}

To date, many works have been reported on the intensity modulation based FODSs (Bock et al., 2001; Cui et al., 2008; Saunders \& Scully, 2007; Miclos \& Zisu, 2001; Kulchin et al., 2007), which the probe consists of a pair of fibers used for transmitting and receiving the light. For instance, Buchade and Shaligram, et al. (2006) presented a FODS using two fibers inclined with a same angular angle and reported the sensitivity was enhanced compared with the conventional sensor with parallel bundled fibers. It also reported that the performances of the FODS with two fibers are depended mainly on four parameters: the offset, the lateral separation and the angle between the transmitting and receiving fiber tips, and the angle of the reflector (Buchade \& Shaligram, 2007). However, there is still a lack of research work on the FODSs with different geometry of the receiving fiber. Hence, a study of this type of FODS is depicted in this section theoretically and experimentally.

\section{Theoretical}

Fig. 13 shows the geometry of the inclined displacement sensor, which consists of a transmitting fiber, receiving fiber and a reflector. The sensor performance is studied at various core radiuses of transmitting and receiving fiber. Fig. 13 (a) (Fig. 13(b)) shows the 
geometry of the sensor in case of the receiving core is bigger (smaller) than the transmitting core. Two asymmetrical transmitting and receiving fibers are mounted at an angle ' $\theta$ ' with reference to the normal to the reflector. This ensures the receiving fiber core to collect the maximum power from cone of emitting light of the transmitting fiber. The shortest distances between the sensor probe tips and reflector are $x_{1}$ and $x_{2}$ for transmitting fiber and receiving fiber, respectively. The dash lines in receiving fiber is represented the size of diameter value same as the transmitting fiber. The image of transmitting (receiving) fiber is formed at a further distance $x_{1}\left(x_{2}\right)$ opposite to the transmitting (receiving) fiber beyond the reflector. The image fiber is thus seen located at $2 x_{1}$ or $2 x_{2}$ from the original position of the probe. Effectively the reflected light appears to form a cone and reaches the receiving fiber, which is parallel aligned in the cone as shown in Fig. 13.

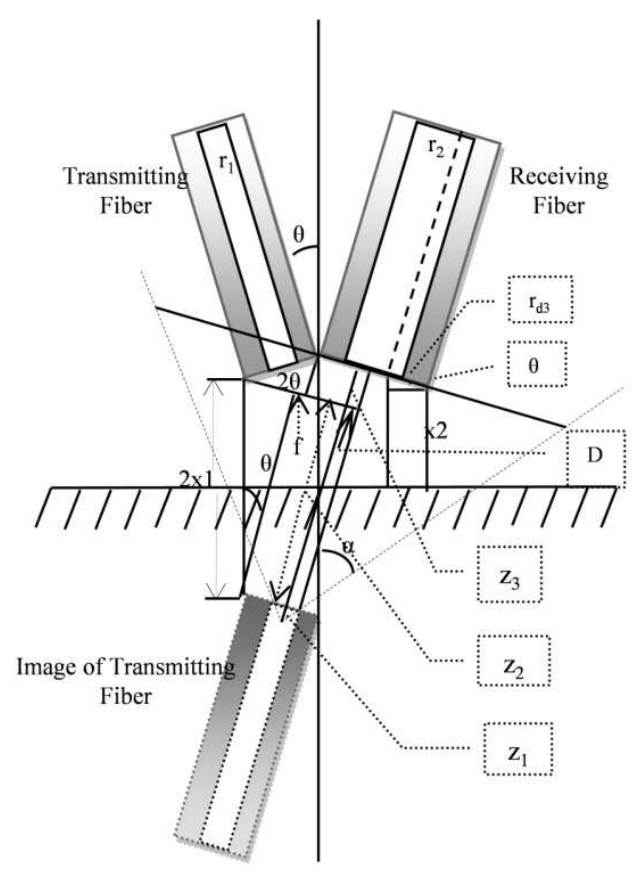

(a)

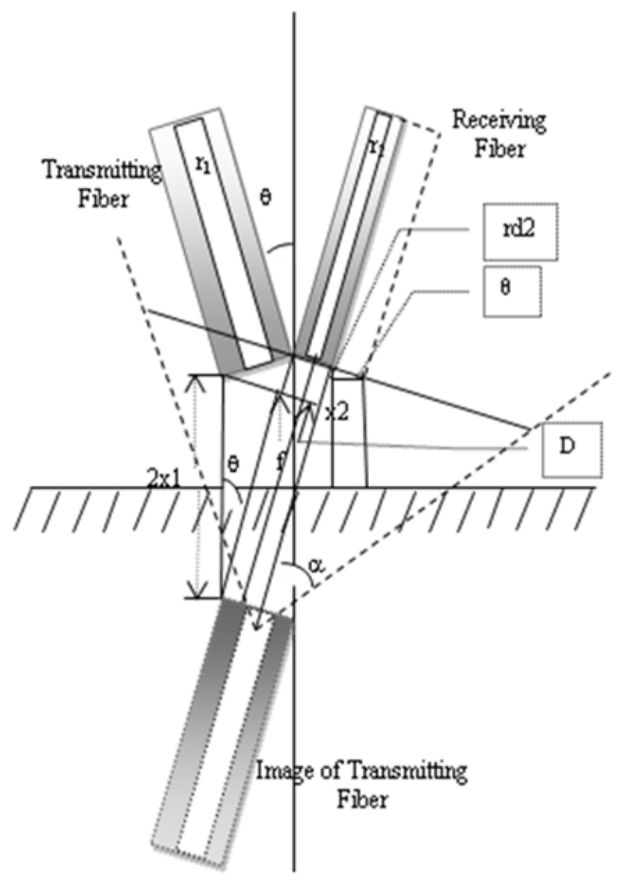

(b)

(a) $\mathrm{r}_{2}>\mathrm{r}_{1}(\mathrm{~b}) \mathrm{r}_{2}<\mathrm{r}_{1}$

Fig. 13. The structure of sensor probes with two asymmetrical inclined fibers

As shown in Fig. 13, the core radius of the transmitting and receiving fibers are denoted as $r_{1}$ and $r_{2}$, respectively. Meanwhile, the diameters of transmitting fiber and receiving fiber are $\mathrm{r}_{\mathrm{d} 1}$ and $r_{d 2}$, respectively. We assume that the ratio between the radius of two fibers is $k_{1}, k_{1}=r_{1} / r_{2}$ and the ratio of diameter of two fibers is $k_{2}=r_{d 1} / r_{d 2}$. From the geometry analysis of Fig. 13, the distance between the two sides of image of transmitting and receiving fibers is given by:

$$
f=\left|r_{d 1} \times \cos (2 \theta)-2 x_{1} \times \sin (\theta)\right|
$$


Then, the distance between two fiber core, $D$ is obtained as;

$$
D=f+\frac{r_{d 2}-r_{d 1}}{2}=f+\frac{r_{d 2}\left(1-k_{2}\right)}{2}
$$

The acceptance angle of transmitting fiber $\mathrm{a}$ is given by $\alpha=\sin ^{-1}\left(\frac{N A}{n}\right)$, where $N A$ is a numerical aperture for the transmitting fiber. The distance between emitting point of transmitted light to the receiving flat core is denoted as $z$, which is given by;

$$
\mathrm{z}=\mathrm{z}_{1}+\mathrm{z}_{2}+\mathrm{z}_{3}
$$

where $z_{1}=r_{1} \times \cot a=k_{1} r_{2} \times \operatorname{cota}, z_{2}=2 x_{1} \times \cos (\theta)$ and $z_{3}=r_{d 1} \times \sin (2 \theta)=k_{2} r_{d 2} \times \sin (2 \theta)$ as illustrated in Fig. 13.

To analyze the power collected by the receiving fiber, we simply analysis the light inside the fiber by using a Gaussion beam approach. The irradiance of emitted light is obeying an exponential law according to Eq. (3). The optical power received by the receiving fiber can be evaluated by integrating the irradiance, I over the surface area of the receiving fiber end which is given by Eq. (4). To simulate conveniently, the Eq. (4) can be described in other expressions;

$$
P_{1}\left(k_{1}, k_{2}, z\right)=\frac{2 P_{E}}{\pi \omega^{2}(z)} \int_{y=-r_{2}}^{r_{2}} \int_{x=D-\sqrt{r_{2}^{2}-y^{2}}}^{D+\sqrt{r_{2}^{2}-y^{2}}} \exp \left(-\frac{2\left(x^{2}+y^{2}\right)}{\omega^{2}(z)}\right) d x d y
$$

The $P\left(k_{1}, k_{2}, z\right)$ is the power collected by the receiving fiber corresponding the parameters $k_{1}$ and $k_{2}$. The radial coordinate $r$ is expressed by $\sqrt{x^{2}+y^{2}}$ in Cartesian coordinate system.

Fig. 14 illustrates the overlap area of the reflected light area and the core of the receiving fiber. The overlap area is zero at $x_{2}=0$ (Fig. 13(a)) or $x_{1}=0$ (Fig. 13(b)) and at a very small displacement (blind area) where the jacket of the two fibers blocks the reflected. As the displacement is increased further, the overlap area increases and thus increases the total power collected by the receiving core. The total power is maxima when the receiving cone covers the entire receiving core area. After that, the received optical power starts to decay exponentially as the displacement continues to increase. The received optical power is strongly dependent on the core size of the receiving fiber. At inclination angle of $2 \theta$ between the transmitting and receiving fibers, the distance $x_{1}$ between the sensor probe tip and reflector is given by (Buchade \& Shaligram, 2006)

$$
x_{1}=r d_{1}(\operatorname{cosec} \theta-2 \sin \theta) / 2
$$

From the geometrical analysis of Fig. 13, the distance $x_{2}$ is obtained as; $x_{2}=x_{1}-r_{d 3} \sin \theta$ for $r_{d 1}<$ $r_{d 2}$ (Fig. 13(a)) or $x_{2}=x_{1}+r_{d 3} \sin \theta$ for $\mathrm{r}_{\mathrm{d} 1} \geq \mathrm{r}_{\mathrm{d} 2}$ (Fig. 13(b)) where $r_{d 3}=r_{d 2}-r_{d 1}$. Therefore, the distance between sensor probe tip and reflector mirror can be summarized as;

$$
x_{2}=\frac{r_{d 2}}{2}\left(k_{2} \operatorname{cosec} \theta+2 \sin \theta\left(1-2 k_{2}\right)\right) \quad(\mathrm{rd} 1>\mathrm{rd} 2)
$$




$$
\begin{array}{ll}
=\frac{k_{2} r_{d 2}}{2}(\operatorname{cosec} \theta-2 \sin \theta) & (\mathrm{rd} 1=\mathrm{rd} 2) \\
=\frac{r_{d 2}}{2}\left(k_{2} \operatorname{cosec} \theta-2 \sin \theta\right) & (\mathrm{rd} 1<\mathrm{rd} 2)
\end{array}
$$

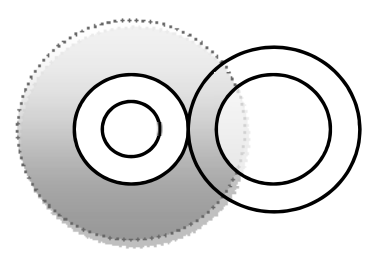

(a)

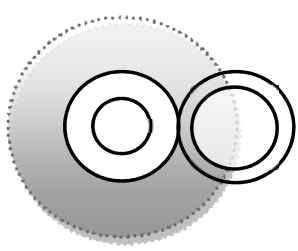

(b)

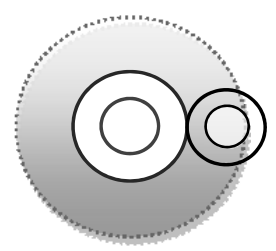

(c)
(a) $r_{d 1}<r_{d 2} ;$
(b) $r_{d 1}=r_{d 2}$; (c)
(c) $r_{d 1}>r_{d 2}$

Fig. 14. Overlap area view

The proposed sensor is simulated by using a MATLAB programming. To simplify the analysis, the $k_{1}$ values of $0.5,0.667,1,1.5$ and 2 are used. The $k_{2}$ value is set based on the availability of the fiber in our laboratory. In this simulation, the $k_{2}$ values of $0.5,1$ and 2 are used. The wavelength of the laser source $\lambda$ is set at $594 \mathrm{~nm}$. The numerical aperture values $\mathrm{NA}_{1}=0.27, \mathrm{NA}_{2}=0.32$ and $\mathrm{NA}_{3}=0.4$ are used for the core radius of $0.25 \mathrm{~mm}, 0.5 \mathrm{~mm}$ and $0.75 \mathrm{~mm}$, respectively.

\section{Experiment}

To verify the simulated results the FODS is constructed by mounting the transmitting and receiving fibers on the plastic board at angle with reference to the normal of the reflector as shown in Fig. 15. Separate samples with various fiber diameters and core radius are prepared for angle $=10^{\circ}, 20^{\circ}$ and $30^{\circ}$. Light from $594 \mathrm{~nm}$ He-Ne laser is modulated by an external chopper at frequency of $200 \mathrm{~Hz}$ and launched into the transmitting fiber. The light has an average output power of $3.0 \mathrm{~mW}$, beam diameter of $0.75 \mathrm{~mm}$ and beam divergence of 0.92 mRads. The length of transmitting and receiving fiber length is approximately $2 \mathrm{~m}$. The transmitting fiber radiates the modulated light from the light source to the target mirror, the displacement of sensor probe tip between mirror is controlled by a piezoelectric \& driver. The reflective light from target mirror, which is mounted in the bottom of tank, is collected by the receiving fiber whose carriers the light into the silicon detector. A lock-in amplifier is connected with the detector to deduce the dc drift. The initial experiment is carried out by varying the inclination angle between the fibers.

\section{Results and discussion}

Fig. 16 compares the experimental and theoretical plots of the normalized output collected against normalized displacement between probe and reflector with air medium in between. In this study, the ratios $k_{1}, k_{2}$, and angle $\theta$ are set at $0.667,0.5,10^{\circ}$ respectively. As shown in the figure, the theoretical curve is in good agreement with the experimental curve, verifying 


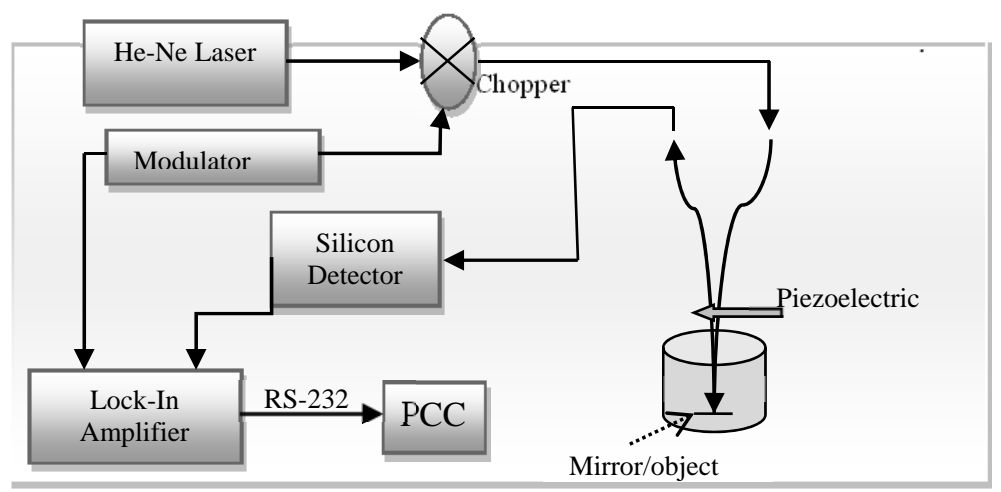

Fig. 15. Experiment setup of proposed FODS with two inclined asymmetrical fibers (Yang, et al., 2009).

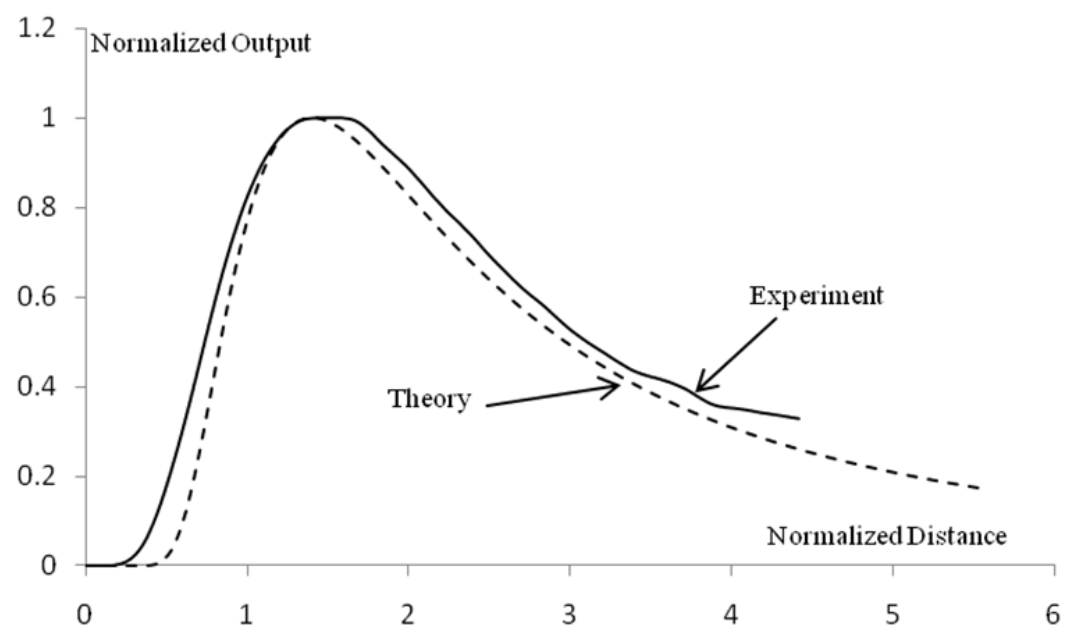

Fig. 16. Comparison between theoretical and experimental curves of the FODS with air medium in between the gap

the feasibility of our theoretical model. It is also observed that up to $0.3(0.5)$ of separation for experimental (theoretical), light in transmitting fiber would be reflected back into itself and little or no light would be transferred to receiving fiber. This is then referred to as the blind region. As the distance increases, the reflected cone overlaps the receiving fiber core and hence the output intensity increases. This relation is continued until the entire face of receiving fiber is illuminated with the reflected light. This point is called optical peak and corresponds to maximum voltage. As the gap increases beyond this transition region, the intensity drops off following roughly an inverse-square law. The small discrepancy between the theoretical and experimental results is due to the noise sources such as shot noise and thermal noise, which are added to the value of the experimental results and are not calculated in theoretical analysis. 
The experiments are also carried out to study the effect of $k_{1}$ and $k_{2}$ values as well as angle $\theta$ on the performance of FODS. Fig. 17 shows the normalized output power against displacement for the FODS at various $k_{1}, k_{2}$ and angle. Figs. 17 (a), (b) and (c) show the curves at $10^{\circ}, 20^{\circ}$ and $30^{\circ}$ respectively with an air gap in between the displacement. By comparing the curves in Fig. 17, we understand that the performance of FODS is strongly depended on the fiber core size. The output power collected by receiving fiber is highest when the $k_{1}$ and $k_{2}$ values are set at 0.667 and 1, respectively. The inclination angle $\theta$ of two asymmetrical fiber core is also effected the sensor performance with the bigger inclined angle has a higher output sensitivity with a lower linearity range. Compared to the FODS with zero inclination angle, the sensitivity of the proposed sensor increased by 3.6, 8.5 and 16 times with the inclination angles of $10^{\circ}, 20^{\circ}$ and $30^{\circ}$, respectively. However, the corresponding linear range reduced by $67 \%, 55 \%$ and $33 \%$, respectively. The performances of the proposed FODS are summarized as shown in Table 3.8. By using the $k_{1}$ and $k_{2}$ values of $(0.667,1)$, the maximum sensitivities of $0.2752 \mathrm{mV} / \mathrm{mm}, 0.3759 \mathrm{mV} / \mathrm{mm}$ and 0.7286 $\mathrm{mV} / \mathrm{mm}$ are obtained at inclination angles of $10^{\circ}, 20^{\circ}$ and $30^{\circ}$, respectively. This sensitivity is higher compared to the previous work by Buchade and Shaligram (2006). The maximum linear ranges of $10.4 \mathrm{~mm}, 7 \mathrm{~mm}$ and $3 \mathrm{~mm}$ are obtained at inclination angles of $10^{\circ}, 20^{\circ}$ and $30^{\circ}$, respectively for the FODS with $k_{1}$ and $k_{2}$ values of $(0.667,1)$.

\begin{tabular}{|c|c|c|c|c|c|c|}
\hline \multirow{2}{*}{ Methods } & \multicolumn{6}{|c|}{ Front slopes } \\
\cline { 2 - 7 } & \multicolumn{2}{|c|}{ Sensitivity $(\mathrm{mV} / \mathrm{mm})$} & \multicolumn{2}{c|}{ Linearity Range $(\mathrm{mm})$} \\
\hline \hline$\left(k_{1}, k_{2}\right)$ & $10^{\circ}$ & $20^{\circ}$ & $30^{\circ}$ & $10^{\circ}$ & $20^{\circ}$ & $30^{\circ}$ \\
\hline$(0.5,0.5)$ & 0.1345 & 0.1838 & 0.4761 & $1.5-3.5$ & $0.4-2.1$ & $0-0.7$ \\
\hline$(0.667,1)$ & 0.2752 & 0.3759 & 0.7286 & $1-5.2$ & $0.1-2.8$ & $0-1$ \\
\hline$(1,1)$ & 0.1671 & 0.2224 & 0.5528 & $1.2-4.8$ & $0.2-2.8$ & $0-1$ \\
\hline$(1.5,1)$ & 0.1885 & 0.2745 & 0.6371 & $1.2-4.8$ & $0.1-2.9$ & $0-1$ \\
\hline$(2,2)$ & 0.0645 & 0.1201 & 0.1904 & $1.4-3.5$ & $0.2-2$ & $0-0.8$ \\
\hline \multirow{2}{*yyyyyyy}{} & & & Back slopes & & \\
\hline$\left(k_{1}, k_{2}\right)$ & $10^{\circ}$ & $20^{\circ}$ & $30^{\circ}$ & $10^{\circ}$ & $20^{\circ}$ & $30^{\circ}$ \\
\hline$(0.5,0.5)$ & 0.0223 & 0.0479 & 0.1447 & $4.3-11.5$ & $2.9-8.9$ & $1.5-3.7$ \\
\hline$(0.667,1)$ & 0.0675 & 0.1336 & 0.3224 & $6.6-17$ & $3.2-10.2$ & $1.3-4.3$ \\
\hline$(1,1)$ & 0.0472 & 0.0823 & 0.2296 & $5.6-14.8$ & $3-9$ & $1.2-4.3$ \\
\hline$(1.5,1)$ & 0.056 & 0.1155 & 0.2929 & $6.8-16$ & $3.2-9.2$ & $1.2-4.3$ \\
\hline$(2,2)$ & 0.0128 & 0.0389 & 0.0885 & $4.5-11.5$ & $2.4-7.5$ & $1-3$ \\
\hline
\end{tabular}

Table 6 . The performances comparison of FODS with two asymmetrical inclined fibers 


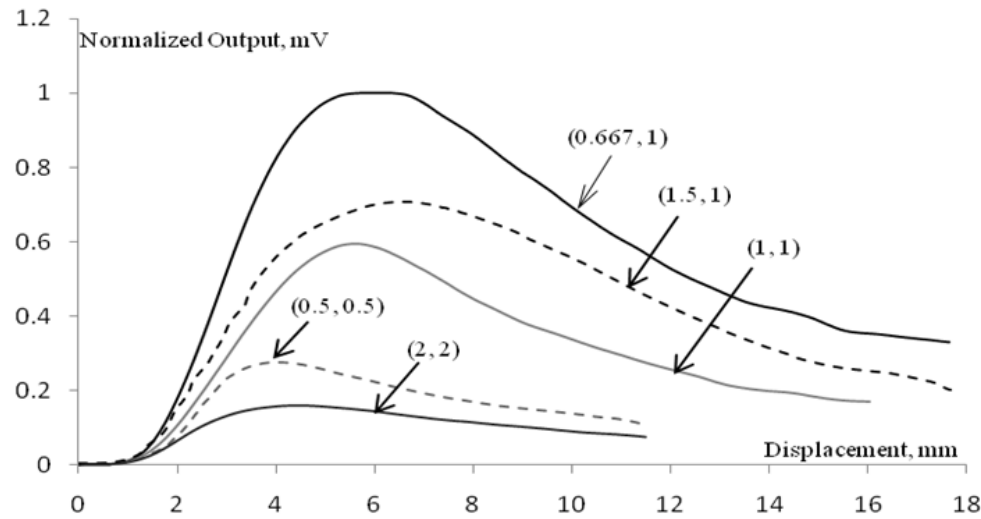

(a) Inclination angle $10^{\circ}$

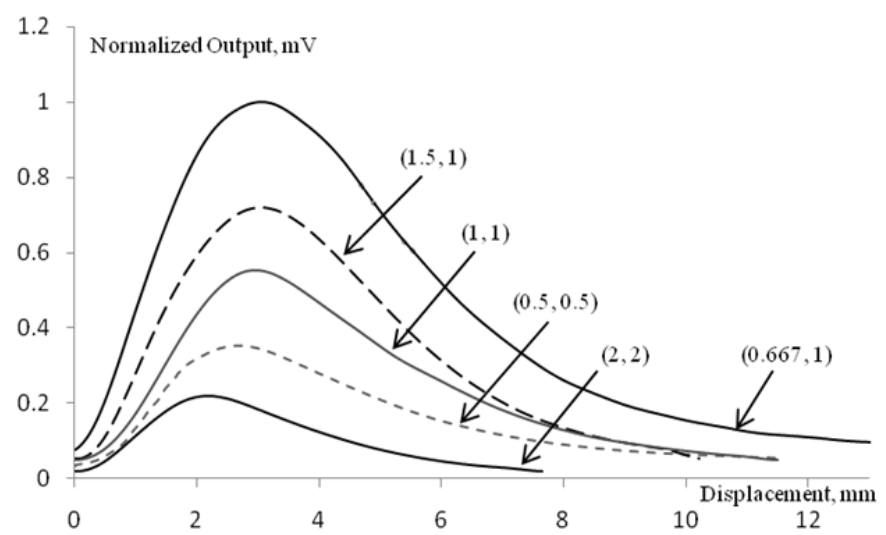

(b) Inclination angle $20^{\circ}$

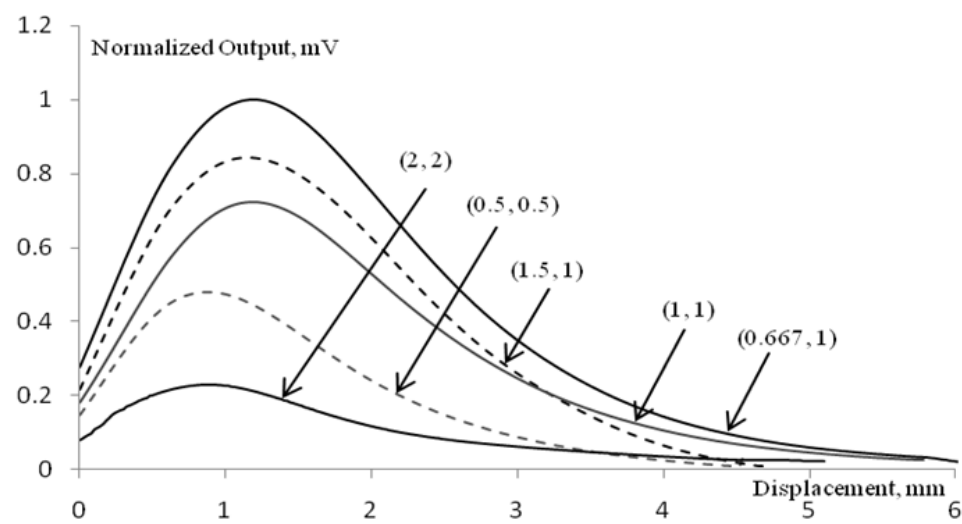

(c) Inclination angle $30^{\circ}$

Fig. 17. The performance output of k1, k2: $(0.667,1),(1,1),(1.5,1),(0.5,0.5)$ and $(2,2)$ in inclined angle (a) $10^{\circ}$ (b) $20^{\circ}$ (c) $30^{\circ}$ 


\section{Applications of the FODS}

Beside displacement measurement, the FODS can also be used to sense many other parameters such as temperature, pressure, refractive index, strain, mass and etc (Bechwith, 2000; Chang et al., 2008; Arellano-Sotelo, 2008). In this section, two different applications of FODS will be described.

\subsection{Liquid refractive index detection using a FODS}

In this study, the sensor probe is containing two pieces of fiber optics, one set connected to a light source and termed the transmitting fiber, and the other set connected to a photo detector (photodiode) and known as the receiving fiber. These two groups of fibers are bundled into a common probe to be used in a FODS. The FODS has a capability to measure physical quantities such as the displacement, vibration, strain, pressure, etc (Yasin et al., 2008; Yasin et al., 2009; Rastogi, 1997). However, the use of FODS sensors for detection of environmental refractive index change has not been fully explored. Refractive index sensing is important for biological and chemical applications since a number of substances can be detected through measurements of the refractive index. In the development of a liquid refractive index sensor (LRIS) (Suhadolnik et al., 1995; Chaudari and Shaligram, 2002; Yang et al., 2009; Nath et al., 2008; Kleizal \& Verkelis, 2007), an intensity modulation in conjunction with multimode plastic fiber is the most suitable technique because of its non-contact sensing and many advantage properties are inherited by the multimode plastic fiber such as efficient signal coupling and being able to receive the maximum reflected light from target.

A FODS based refractive index measurement using a bundle fiber is first introduced by Suhadolnik et al. in 1995. Later on Chaudhari \& Shaligram reported on study of LRIS at various types of optical sources. In our earlier work, a FODS was proposed based on two asymmetrical fibers for liquid refractive index measurement (Yang et al., 2009). In this section, a new LRIS is studied and demonstrated by using pair type of fibers bundled at various inclination angles.

The structure of proposed LRIS is shown in Fig. 18, which consists of a pair of transmitting and receiving fiber. We assume that the transmitting and receiving fibers have inclination angles of $\theta_{1}$ and $\theta_{2}$, respectively against the y-axis. The image of transmitting fiber is located opposite of the mirror with same distance. The central of the receiving fiber and the image of transmitting fiber are pointed as $O^{\prime}$ and $O$, respectively in Fig. 18. From the geometrical analysis of Fig. 18, the angle $\alpha=\sin ^{-1}(N A / n)$ and $z_{a}=\frac{r_{1}}{\tan \alpha}$. Therefore, the following distances are given by;

$$
\begin{gathered}
A B=\sqrt{z_{a}^{2}+r_{d 1}^{2}} \sin \left(\tan ^{-1}\left(\frac{r_{d 1}}{z_{a}}\right)-\frac{\pi}{2}+\theta_{1}\right), \\
O^{\prime} C=4 r_{d 1} \sin \theta_{1}+2 x-A B-r_{d 2} \sin \theta_{2}, \\
O A=\sqrt{z_{a}^{2}+r_{d 1}^{2}} \cos \left(\tan ^{-1}\left(\frac{r_{d 1}}{z_{a}}\right)-\frac{\pi}{2}+\theta_{1}\right)
\end{gathered}
$$




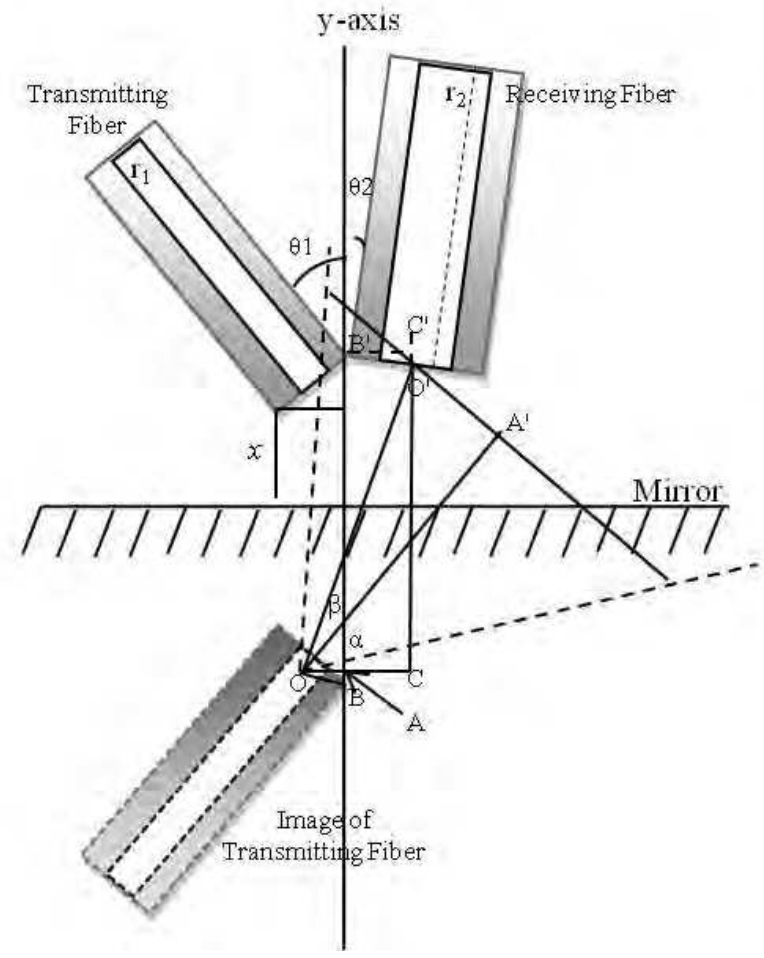

Fig. 18. Structure of sensor probe for the sensing of liquid refractive index.

$$
\begin{gathered}
O C=O A+r_{d 2} \cos \theta_{2} \\
O O^{\prime}=\sqrt{O^{\prime} C^{2}+O C^{2}}
\end{gathered}
$$

where the NA is numerical aperture of transmitting fiber, $\mathrm{n}$ is refractive index of liquid, $\mathrm{r}_{1}$ and $r_{2}$ are the core radius of transmitting fiber and receiving fiber while the $r_{d 1}$ and $r_{d 2}$ are the radius of transmitting fiber and receiving fiber, respectively and the $\mathrm{x}$ is the displacement between the sensor probe tip and reflector mirror.

Also from the geometrical analysis, the acceptance angle $\beta$ of the light cone from the virtual point source $O$, is given by

$$
\beta(z)=\tan ^{-1}\left(\frac{O^{\prime} C}{O C}\right)-\frac{\pi}{2}+\theta_{1}
$$

The intensity of the light emitted from the transmitting fiber can be well described with Gaussian distribution (Chang, et al., 2008) and is given by;

$$
I(r, z)=\frac{2 P_{E}}{\pi \omega^{2}(z)} \exp \left(-\frac{2 r^{2}}{\omega^{2}(z)}\right)
$$


where $r$ is the radial coordinate, $z$ is the longitudinal coordinate. $\omega(z)$ is the beam radius and expressed as a function of $z, \omega(z)=\omega_{0} \sqrt{1+\left(\frac{z}{z_{R}}\right)^{2}}$. The waist radius $\omega_{0}$ and Rayleigh range $z_{R}$ are the important parameters in the Gaussian Beam function and the detailed description can be found (Chang et al., 2008). Eq. (30) shows that the light intensity decays exponentially as it goes radially away from the center of the light circle. The radial coordinate $r$ of Eq. (30) can be determined by;

$$
r=O O^{\prime} \sin \beta
$$

The longitudinal coordinate is the distance between the sensor probe tip and the virtual laser source point $O$ and it can be determined

$$
z=O O^{\prime} \cos \beta
$$

For points situated in the far-field $z>>z_{R}$, the beam radius of the virtual point source can be derived as

$$
\omega(z) \approx z \alpha
$$

By the approximation,

$$
r_{1}=z_{a} \tan \alpha \approx z_{a} \alpha
$$

Based on the properties above, the power harnessed by the receiving fiber, $P$ can be evaluated by integrating the Gaussian distribution function of Eq. (30) over the area of the of receiving fiber end surface, $S_{r}$

$$
P(r, z)=\int_{S_{r}} I(r, z) d S_{r}
$$

where the core area of the receiving fiber is

$$
S_{r}=\pi r_{1}^{2}=\pi z_{a}^{2} \alpha^{2}
$$

By combining and substituting Eqs. (31), (32), (33) and (36) into the Eq. (35), finally the proposed LRIS response can be summarized as;

$$
P_{(z, r)}=\frac{2 P_{E}}{\pi \omega^{2}(z)} \exp \left(-\frac{2 r^{2}}{\omega^{2}(z)}\right) \times S_{r}=\frac{2 z_{a}^{2} P_{E}}{z^{2}} \exp \left(\frac{2 r^{2}}{z^{2} \alpha^{2}}\right)
$$

This equation shows that the liquid refractive index response of sensor is a function of displacement $x$ and refractive index $n$ of surrounding medium while sensor probe is design of inclination angles of $\theta_{1}$ and $\theta_{2}$. Therefore, based on Eq. (37), the proposed LRIS can be used to detect the liquid refractive index where the sensor probe is immersed by the liquid to be measured.

The mathematical model of proposed LRIS is simulated by MATLAB programming. In the simulation, the wavelength of the laser source $\lambda$ and numerical aperture $N A$ is set at $594 \mathrm{~nm}$ 
and 0.32 , respectively. The fiber core radius $r_{1}$ and $r_{2}$ are set at $0.25 \mathrm{~mm}$ and $0.5 \mathrm{~mm}$ while the fiber diameters $r_{d 1}$ and $r_{d 2}$ are set at $0.5 \mathrm{~mm}$ and $0.75 \mathrm{~mm}$, respectively. Fig. 19 shows the experimental set-up, which consists of a 594nm yellow He-Ne laser as a light source and a bundled fiber as a probe. The emitted laser light has an average output power of $3.0 \mathrm{~mW}$, beam diameter of $0.75 \mathrm{~mm}$ and beam divergence of $0.92 \mathrm{mRads}$. The external chopper modulates the light at a frequency of $200 \mathrm{~Hz}$ before it is launched into the transmitting fiber. The transmitting fiber transfers the modulated light from laser source to reflector mirror while the receiving fiber collects the reflection light to the detector. The sensor probe is mounted on the stage controlled by NewFocus Picomotor for the displacement measurement. Silicon detector measures the received light and converts it into electrical signal which is then denoised using the lock-in amplifier. During the measurement, the room temperature is keeping in $28^{\circ} \mathrm{C}$ to avoid the change of liquid refractive index.

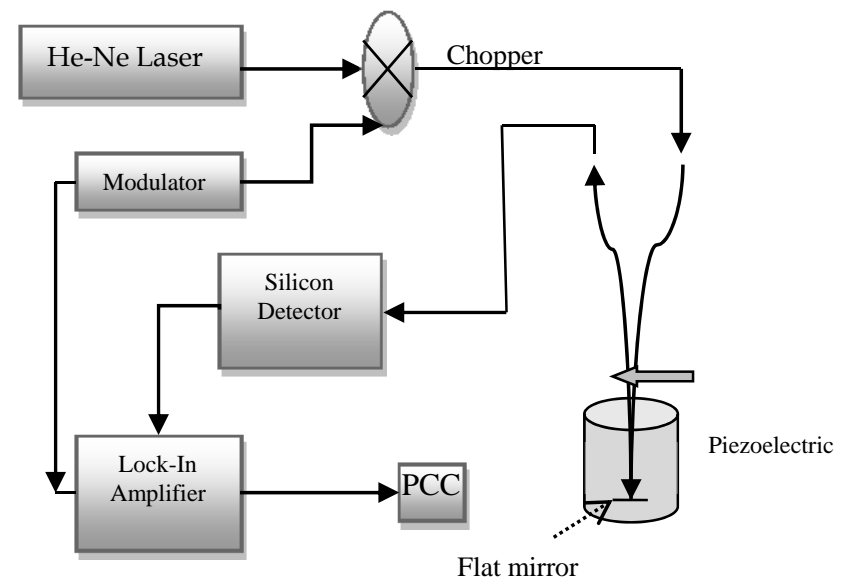

Fig. 19. Experimental set-up of the proposed liquid refractive index sensor

The simulation results are illustrated in Fig. 20. This figure shows the relationship between the sensor responses and probe inclination angles $\theta_{1}$ and $\theta_{2}$ in the measurement of three different refractive indices 1, 1.3 and 1.6 based on the probe inclined with the same angles of $0,10^{\circ}$ and $20^{\circ}$. In Fig. 5.8, the outputs powers are normalized in 1 and the displacements are simulated in $\mathrm{mm}$. Three different group curves are showing three various sensors response based on the three various inclination angles. As seen in Fig. 20, it was found that the inclination angles $\theta_{1}$ and $\theta_{2}$ are reasonably affects the displacement curves profile and output power. The highest output power is almost 10 times of the lowest output power. The vertical dash lines are located in the displacements of $1.1 \mathrm{~mm}, 2.0 \mathrm{~mm}$ and $3.4 \mathrm{~mm}$ corresponding to the sensor probe inclination angles $20^{\circ}, 10^{\circ}$ and 0 , respectively. In those positions, the sensor responses have the biggest output differentiation in the increase of refractive indices from 1.0 to 1.5. At those positions, the sensor output intensity increases almost linearly with the increase of the refractive index of the medium. From Fig. 20, we can observe that the increase the inclination angles improves the performance of the LRIS. The larger the inclination angles of $\theta_{1}$ and $\theta_{2}$, the better the performance of liquid refractive index response. 


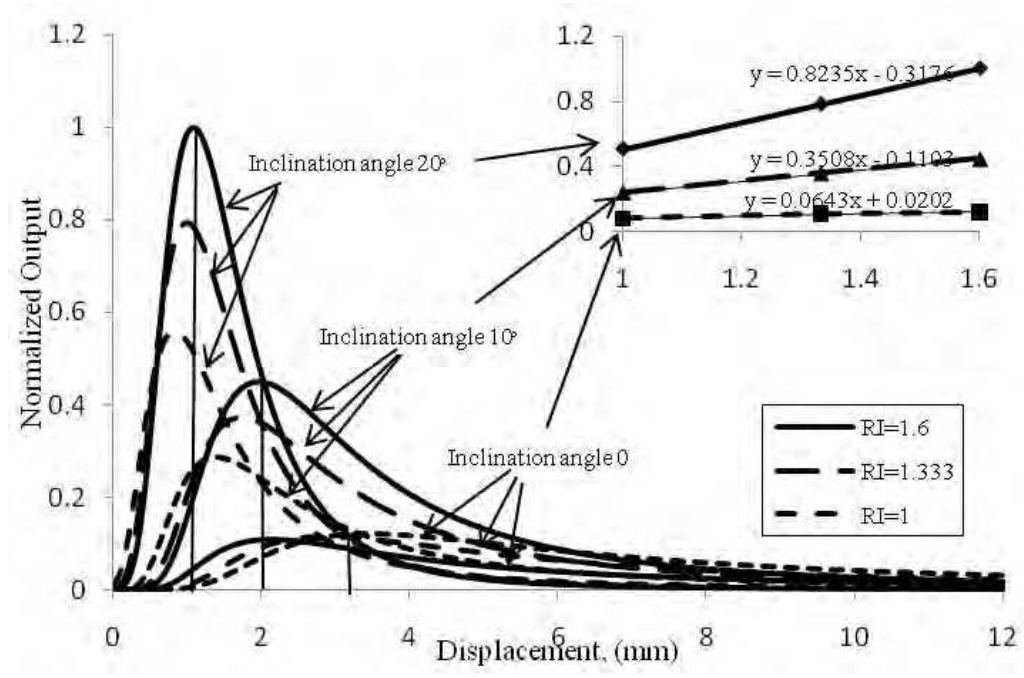

Fig. 20. Simulation results for the displacement at various inclination angles and refractive indices. Inset shows the maximum normalized output against the refractive index for inclination angles of $0^{\circ}, 10^{\circ}$ and $20^{\circ}$.

Inset of Fig. 20 shows the maximum normalized output of the sensor as a function of refractive index for various inclination angles. The normalized outputs were taken at the sensor probe positions of $1.1 \mathrm{~mm}, 2.0 \mathrm{~mm}$ and $3.4 \mathrm{~mm}$ for inclination angles of $20^{\circ}, 10^{\circ}$ and $0^{\circ}$, respectively, which is indicated by vertical dash lines in Fig. 20. It was found that the sensitivities of the sensor increases with the increment of probe inclination angle. As shown in the inset of Fig. 20, the highest sensitivity of 0.8235 is achieved by the use of probe with inclination angle of $20^{\circ}$ which is almost 13 times higher than that in zero inclination. Fig. 21 shows the simulation curves of the LRIS at various inclination angles for the receiving and transmitting fibers when the refractive index of liquid is set at 1.3. It is clearly seen that the inclination angle of receiving fiber $\theta_{1}$ has the stronger affect in the sensor output compared with the angle $\theta_{2}$. As shown in Fig. 21, the highest output power is achieved by the inclination angles; $\theta_{1}=20^{\circ}$ and $\theta_{2}=10^{\circ}$. The lowest output power is observed when the inclination angles of $\theta_{1}$ and $\theta_{2}$ are set at $0^{\circ}$ and $10^{\circ}$, respectively. These results show that the sensor sensitivity can be increased by increasing the inclination angle especially for $\theta_{1}$. However, increasing the inclination is very difficult to be implemented in the experiment unless we can control the position of both fibers very precisely.

In our experiment, three different liquids: isopropyl alcohol, water and methanol are used as the surrounding medium at two conditions; zero inclination for both fibers and the same inclination angles of $10^{\circ}$ for both fibers. The refractive index values for isopropyl alcohol, water and methanol are 1.377, 1.333 and 1.329, respectively. The sensor performance with air surrounding medium is also carried out for comparison purpose. During the experiment operation, the sensor probe is mounted onto the stage and the tank is fixed in the experiment table. The liquid in the tank is changed without moving the tank to ensure the accuracy of the measurement. The room temperature was kept at $28^{\circ}$ to ensure that the refractive index of the liquid is maintained and only displacement parameter is changed in the experiment. 


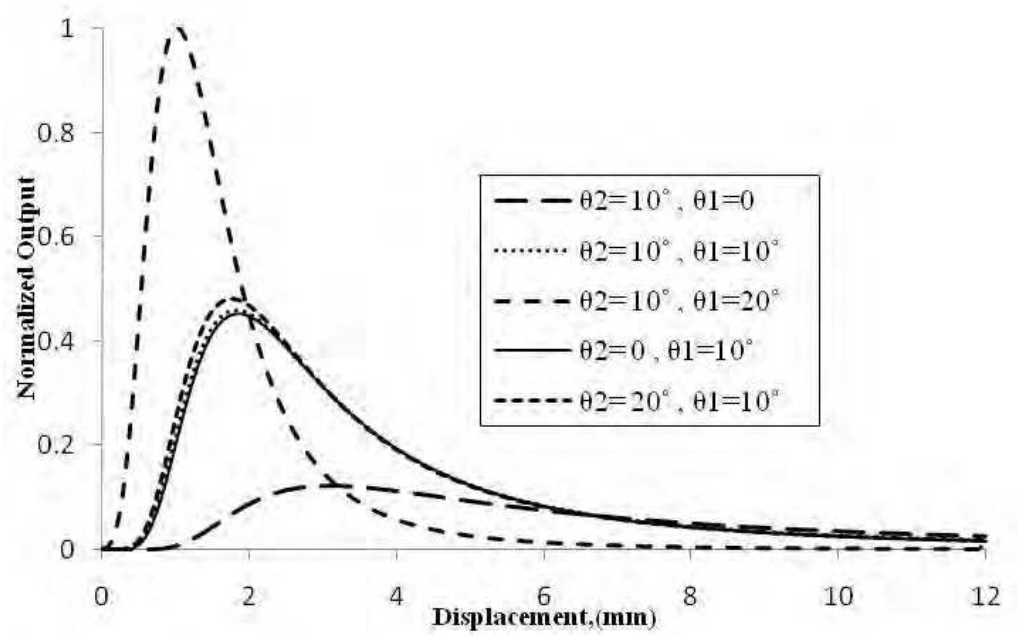

Fig. 21. The output power against displacement for various probe inclination angle combinations when the refractive index is set at 1.3.

Fig. 22 shows the displacement curve at various surrounding media when the inclination angles are set at $0^{\circ}$ for both transmitting and receiving fibers. As shown in this figure, the normalized peak output power increases from 0.83 to 1.00 as the refractive index increases from 1.329 (methanol) to 1.377 (alcohol). It is also found that the displacement position for the peak output increases from 4.0 to $5.1 \mathrm{~mm}$ as the refractive index increases from 1.329 to 1.377. This is attributed the acceptance cone angle that increases with the refractive index increase. The larger acceptance angle provides a mean to collect more signal power. Fig. 23 shows the displacement curves when the inclination angles for both fibers are increased to $10^{\circ}$. As seen in Fig. 23, the peak power and its position increase with refractive index. The peak location of the curve also increases from $3.0 \mathrm{~mm}$ to $3.4 \mathrm{~mm}$ as the refractive index changes from 1.329 to 1.377. From these experimental results, it was found that the sensitivity of the sensor with $10^{\circ}$ inclination of probe arrangement shows a higher sensitivity compared to that of the use of straight probe. The sensitivities are obtained at $0.11 \mathrm{~mm}^{-1}$ and $0.04 \mathrm{~mm}^{-1}$ for the sensors with $10^{\circ}$ and $0^{\circ}$ inclination angles respectively. This finding may be quite useful for chemical, pharmaceutical, biomedical and process control sensing applications.

\subsection{The monitoring of liquid level using a FODS}

Liquid level measurement is vital in many industry areas, finding the applications in such as fuel storage system, chemical processing, etc. Fiber based liquid level sensors are received in prefer of studies since they inherit many merits such as they are non-conductive, antierosion, and immune to electromagnetic interference. In past few years, several technical are employed to develop the fiber optic Liquid Level Sensors (LLSs). Fiber Bragg grating technology was carried out to sense the changing of liquid level by (Yun et al., 2007; Sohn \& Shim, 2009; Dong \& Zhao, 2010). Bending the multimode fiber for multi-points liquid level monitoring can be found in (Lomer et al., 2007). In these cases, the sensing elements are submerged inside the liquid to indicate the presence of liquid. These immersed sensing 


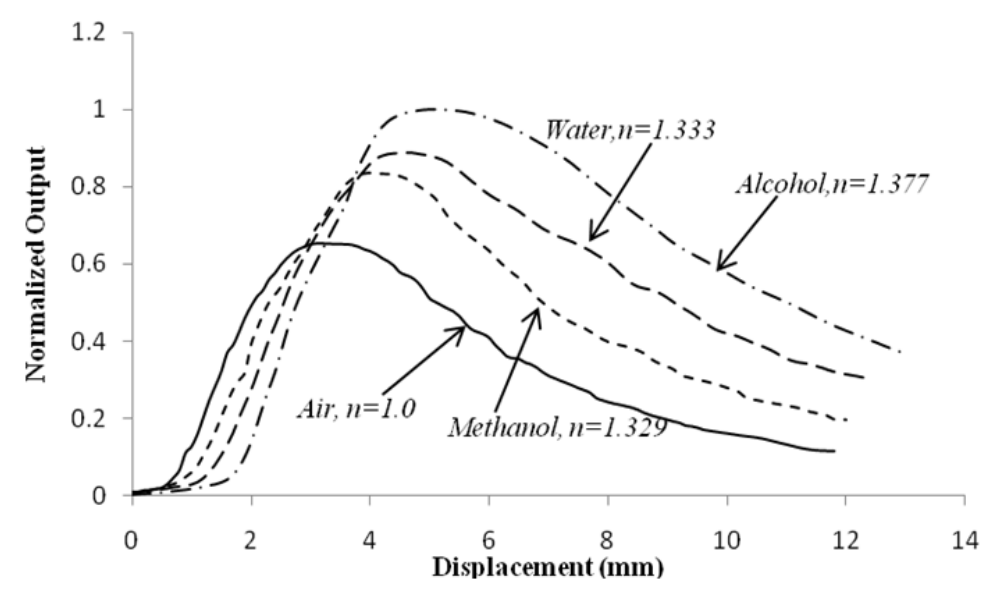

Fig. 22. Experiment results of the displacement curve at various liquid materials when the probe inclination angles are set at $0^{\circ}$.

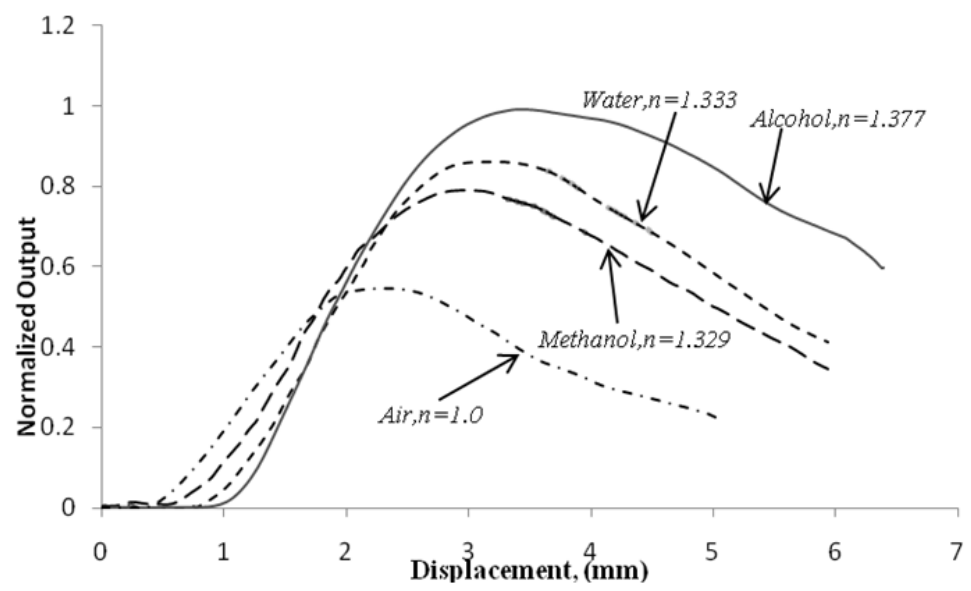

Fig. 23. Experiment results of the displacement curve at various liquid materials when the probe inclination angles for both transmitting and receiving fibers are set at $10^{\circ}$

elements have some limitations in their applications, such as in harsh environments, acid solutions, etc. Thereby, the fiber as sensing element for non-contact the measurement liquid seems good way to explore. There is one proposed sensor (Sohn and Shim, 2009) which employed one fiber bragg grating embedded inside the cantilever beam to connect with a float for indicating the level changing. This case is limited in its cost, surrounded temperature, and sensitivities.

In this section, a simply and cost efficiency intensity based LLS is proposed. A straightforward displacement sensor is employed; its sensing probe is engaged with a float which is contact with the measurement liquid to conduct the information of liquid. This simply setup transfers the sensing of liquid level into the measurement of displacement. It breaks down the limitations of sensitivities and measurement range which can be conquest 
by the selection of displacement sensors. Thus, in this study two type of displacement sensor are used to supply a flexible and compatible sensing of liquid level.

The basic sensing principle is the sensor probe displacement moving that causes from the moving of float during the liquid level change. A schematic setup of proposed fiber optic LLS is illustrated in Fig.24. It composed a He-Ne laser source light wavelength in 594nm (average output power $3.0 \mathrm{~mW}$, beam diameter $0.75 \mathrm{~mm}$ and beam divergence $0.92 \mathrm{mRads}$ ) which coupling the light into the transmitting fiber ( $2 \mathrm{~m}$ length); a external chopper modulates the light at a frequency of $200 \mathrm{~Hz}$ before launched into the transmitting fiber; a concave mirror (or flat mirror) is located at end of transmitting fiber and reflects the guided light of transmitting fiber into a receiving fiber which is bundled with the transmitting fiber. The receiving fiber guides the light into the photo detector which converts the light power into voltage. Meantime, the bundled fiber as sensor probe is fastened by an L cantilever beam which connects a float on the other side. The float is contact with the measurement liquid to indicate the information of liquid levels. A model SR-510 lock-in amplifier is connected with the chopper and photo detector. It plays the function of experiment dataacquisition system, matches the phase between the modulation light and modulator chopper and removes the noise generated by laser source, photo detector and amplifier.

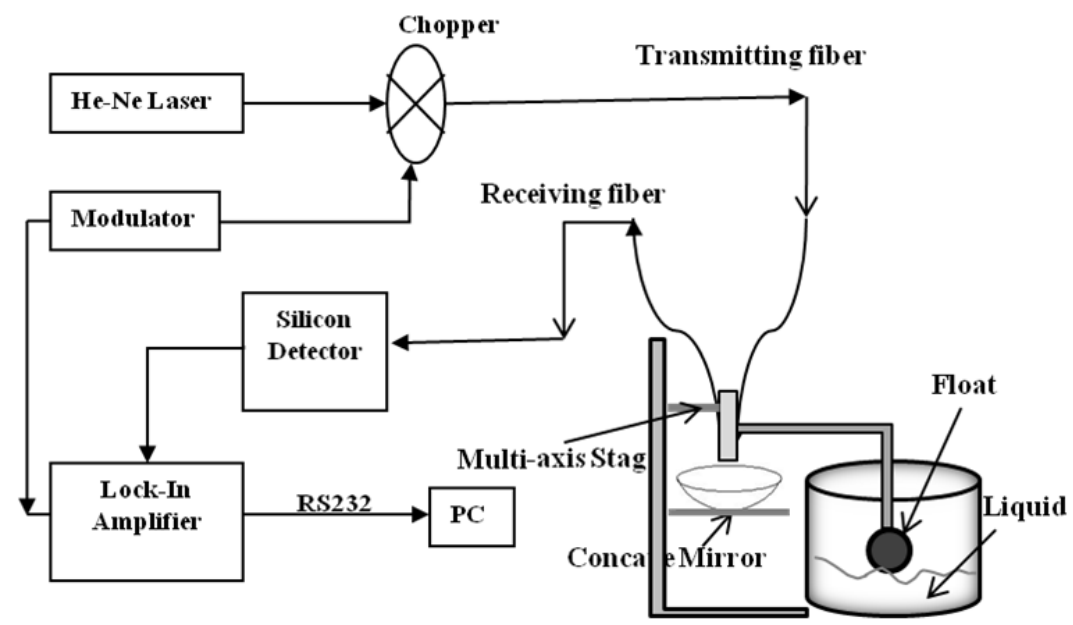

Fig. 24. Experiment setup of monitoring the liquid level

In the using of concave mirror as the reflector, the transfer function of proposed LLS is governed by parameters, namely focal length $f$ and the diameter of the circular concave mirror $D$. The characteristic of the proposed sensor can be found the detailed theoretical analysis in paper (Yang et al., 2010). In consideration of the limited reflecting surface area and FL of the concave mirror, the transfer function of the proposed sensor can be given as

$$
P(u)=\frac{2 z_{a}^{2} P_{E}\left\{1-\exp \left(-\frac{D^{2}}{2\left(u+z_{a}\right)^{2} \theta^{2}}\right)\right\}}{\left[u-\frac{\left(u+z_{a}\right) f}{u-f+z_{a}}\right]^{2}\left(\frac{u+z_{a}}{f}-1\right)^{2}} \exp \left(-\frac{8 z_{a}^{2}}{\left[u-\frac{\left(u+z_{a}\right) f}{u-f+z_{a}}\right]^{2}\left(\frac{u+z_{a}}{f}-1\right)^{2}}\right)
$$


where $P_{E}$ is the emitting power of laser source, $u$ is the distance between the sensor probe tip and concave mirror, $\theta=\sin ^{-1}(N A)$ and $N A$ is the numerical aperture of the transmitting fiber. By using the flat mirror as the reflector, the transfer function of proposed sensor was analyzed in (Lim et al., 2009) detailed. It is given by

$$
P(z)=\frac{2(k+1)^{2}}{\xi^{2}} \exp \left(1-\frac{2(1+k)^{2}}{\xi^{2}}\right)
$$

The core radial ratio $k$ is the ratio of the transmitting fiber core radius to the receiving fiber core radius, $\xi$ is the normalized displacement.

From the transfer functions of Eq. (38) and (39), it can be seen that the sensor response is only influenced by the focal length $f$ and the diameter of the circular concave mirror $D$ in the concave mirror as reflector and by the core radial ratio $k$ for that of flat mirror. As such, the proposed LLS has the widely compatible for the variants type of FODSs, which can escapes the trade-off design in the selection of sensitivity and measurement range. Furthermore, according to the Eqs. (38) and (39), there are no more parameters can affect the sensor response, hence, the proposed LLS is independency for surrounded environments.

Fig. 25 depicts the experiment result when the liquid level is moving upward. In this experiment, the proposed LLS is composed two plastic fiber bundled parallel as sensor probe, a concave mirror with focal length $10 \mathrm{~mm}$ and diameter $24 \mathrm{~mm}$ as reflector. From Fig. 25 , it can be seen that the sensor output intensity is modulated by the moving of liquid level in six variants slops. According to this, hence, the proposed sensor can achieve the continue monitoring of liquid level in the multi-points. Totally six monitoring points are shown in Fig. 25, each point is represented each slop which can be used as the each level of liquid measurement. These six monitoring points are located in the total measurement range of $25 \mathrm{~mm}$, which can provide variants sensitivities and measurement range. The performances of these monitoring points are summarized in table 7.

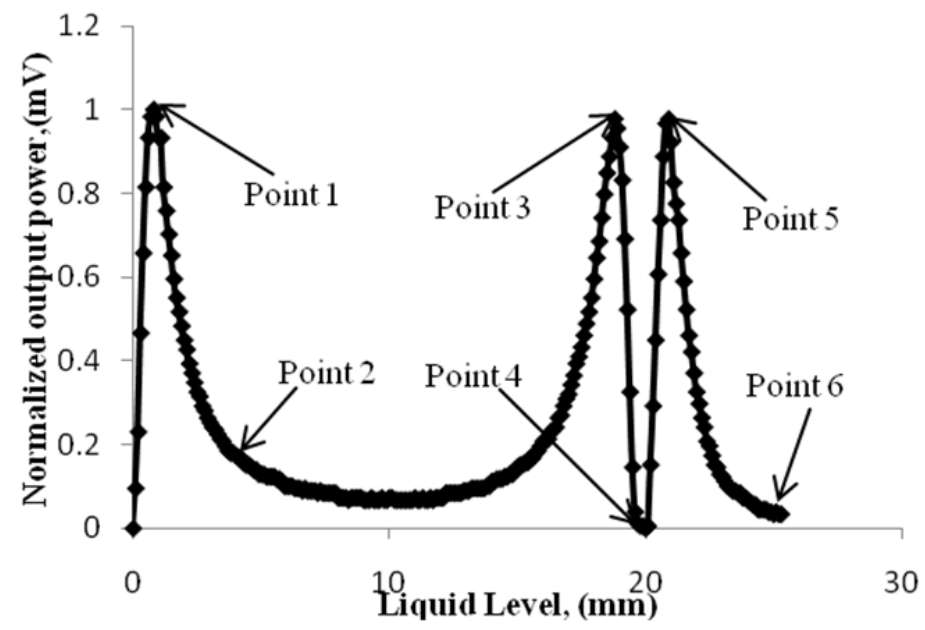

Fig. 25. The experiment result of proposed sensor for concave mirror as reflector and two plastic fibers bundled parallel 
The second experimental is carried out by the sensor probe consisted with two bundled parallel and a flat mirror as reflector. On the other hand, another experimental is repeated by two asymmetrical plastic fiber bundled parallel as sensor probe and a flat mirror as reflector. Both of responses are illustrated in Fig. 26, where each of the curves are displayed two various slops in the measurement range of $3 \mathrm{~mm}$ and $3.5 \mathrm{~mm}$, respectively. From the observation of Fig. 26, two various monitoring points are given from both curves as shown. They are occupied the measurement ranges of $3 \mathrm{~mm}$ and $3.5 \mathrm{~mm}$, respectively. In Fig. 26, the higher sensitivity is obtained from the curve of $k=1$ and the bigger measurement range is supplied by the curve of $k=0.5$. The output curves in Fig. 26 are shifted to the right along the further of liquid level as the value of $k$ decreases. Therefore, the sensitivities of front slope and back slope of the curves are decreasing with decreasing the values of $k$. Mean times, the linearity ranges of curves are enlarged with decreasing the values of $k$. The sensitivities and linearity ranges of Fig. 26 are summarized in Table 7.

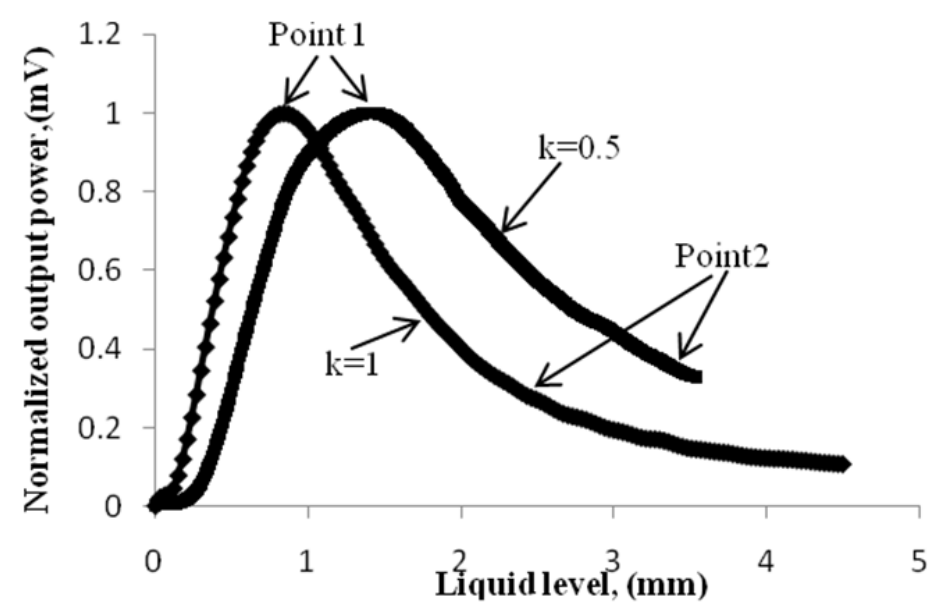

Fig. 26. The experiment results of various ratios of fibers bundled as sensor probe.

Table 7 tabulates the performances of LLS for three different experiments. From the results summarized in table 7 , it can be seen that the highest sensitivity is $1.4533 \mathrm{mV} / \mathrm{mm}$ when its measurement range is $0.84 \mathrm{~mm}$. The largest measurement range $4.8 \mathrm{~mm}$ is obtained from the monitoring point 3 for the concave mirror as reflector. In the experiment of concave mirror as reflector, the monitoring points 1,4 , and 5 have the higher sensitivities and shorter measurement ranges than that of 2,3 , and 6 . In the experiment of flat mirror as reflector, the sensitivities for $\mathrm{k}=0.5$ are decreased by $52.5 \%$ and $14.8 \%$ for of front slope and back slope, respectively, if compared to $\mathrm{k}=1$. The measurement ranges of front slope and back slope are increased by $46.2 \%$ and $18.8 \%$ if compared with the $\mathrm{k}=1$. From the analysis, by using a particular combination of fiber core diameters and reflector, two different $k$ values, ten different sensitivity and linear range selections can be achieved by using the proposed method. Thus, the proposed fiber optic LLS has wide compatibility for other type of displacement sensor to avoid the trade-off selection of sensitivity and measurement range which is always encountered in the design of conventional fiber optic LLS. 


\begin{tabular}{|c|c|c|c|}
\hline Methods & Monitoring point & $\begin{array}{c}\text { Sensitivity } \\
\mathbf{( m V / m m )}\end{array}$ & $\begin{array}{c}\text { Measurement } \\
\text { range (mm) }\end{array}$ \\
\hline \hline \multirow{4}{*}{$\begin{array}{c}\text { Concave mirror } \\
\text { k=1 }\end{array}$} & Point 1 & 1.4026 & $0-0.8$ \\
\cline { 2 - 4 } & Point 2 & 0.2649 & $0.8-3.8$ \\
\cline { 2 - 4 } & Point 3 & 0.1625 & $14-18.8$ \\
\cline { 2 - 4 } & Point 4 & 1.030 & $18.9-20$ \\
\cline { 2 - 4 } & Point 5 & 1.3015 & $20.1-20.9$ \\
\hline \multirow{2}{*}{ Flat mirror k=0.5 } & Point 6 & 0.1821 & $0.1-1.4$ \\
\cline { 2 - 4 } & Point 1 & 0.9529 & $1.4-3.5$ \\
\hline \multirow{2}{*}{ Flat mirror k=1 } & Point 2 & 0.3443 & $0-0.84$ \\
\cline { 2 - 4 } & Point 1 & 1.4533 & $0.84-3$ \\
\hline
\end{tabular}

Table 7. The Sensitivities and measurement ranges of proposed sensor

In the literature of (Sohn and Shim, 2009), the wavelength-shift sensitivity is around $0.15 \mathrm{~nm} / \mathrm{cm}$ and the resolution of intensity modulated the liquid level is approximated $0.1 \mathrm{~dB} / \mathrm{cm}$ are achieved. However, these sensitivities cannot satisfy the high precision measurements and the LLS proposed in (Sohn and Shim, 2009) is high costly. In this paper, the highest sensitivity is achieved by using a probe with two fibers bundled and flat mirror as reflector, it is around 100 times higher than that of (Sohn and Shim, 2009) and is 20 times lower in the cost.

\section{Conclusion}

In this chapter, the performance of various FODSs is investigated theoretically and experimentally. A beam-through FODS has been demonstrated for sensing of lateral and axial displacements, which shows that the usage of smaller core normally shows a better sensitivity and resolution with the expense of the smaller linear range. Various reflective FODSs are then proposed to provide a possibility for the development of the cheap, simple, sensitive and wide dynamic range sensor systems. Compared with the conventional sensor with only one receiving fiber, FODS with two receiving fibers is observed to have a better linearity range. The performance of FODS with asymmetrical bundled fiber is also theoretically and experimentally demonstrated in this chapter. The effect of different core radial ratios (CRRs) on the performance of the sensor is investigated in terms of dynamic range and sensitivity. The experimental results are almost in agreement with the theoretical results. The location of the maximum output is shifted toward the right along the axis of displacement as the value of $k$ increases. Besides, the linear range for both front and back slopes increases with the value of $\mathrm{k}$. To improve the sensitivity, a FODS with two inclined asymmetrical fiber has been proposed. This FODS has been applied in liquid refractive index sensor. An extra-low cost, ultra-high sensitivity, and wide compatibility liquid level fiber optic sensor has been demonstrated as another application for the FODS. A float is touched with the measurement liquid to translate the information of liquid change to the sensor probe displacement moving where an L cantilever beam is fixed in between, the moving of sensor probe finally results the output intensity is modulated. All the theoretical and experimental results are capable of offering quantitative guidance for the design and implementation of a practical FODS. 


\section{References}

Arellano-Sotelo, H.; Barmenkov, Yu. O.; Kir'yanov, A.V. (2008). The use of erbium fiber laser relaxation frequency for sensing refractive index and solute concentration of aqueous solutions. Laser Physics Letters, Vol. 5, No. 11, pp. 825-829.

Bechwith, M.L. (2000). Mechanical measurement. Addison Wesley Longman.

Bergamin; Cavagnero, G.; Mana, G. (1993). A displacement and angle interferometer with subatomic resolution. Rev Sci Instrum, 64, 3076-3081.

Bock, W.J.; Nawrocka, M.S.; Urbanczyk, W. (2001). Highly sensitive fiber optic sensors for dynamic pressure measurement. IEEE Trans. Instrum. Meas., Vol. 50, No. 5, pp. 1085-1088.

Buchade, P.B. \& Shaligram, A.D. (2006). Simulation and experimental studies of inclined two fiber displacement sensor. Sensor and Actuators A, Vol. 128, pp. 312- 316.

Buchade, P.B. \& Shaligram, A.D. (2007). Influence of fiber geometry on the performance of two-fiber displacement sensor. Sensors and Actuators A, 136, 199- 204.

Cao, M.H.; Chen, Y.; Zhou, Z.; Zhang, G. (2007). Theoretical and experimental study on the optical fiber bundle displacement sensors. Sensor and Actuators A, Vol. 136, pp. 580587.

Chang, J.; Wang, Q.; Zhang, X.; Ma, L.; Liu, T.; Wang, Q.; Liu, Z.; Zhang S.; Ding, S. (2008). Single-end vibration sensor based on an over-coupled fiber-loop reflector. Laser Physics, Vol. 18, pp. 452-454.

Chaudhari, A.L. \& Shaligram, A.D. (2002). Multi-wavelength optical fiber liquid refractometry based on intensity modulation", Sensor and Actuators A, Vol.100, pp.160- 164.

Cui, C.; Wang, Q.; Hu, X.; Yu, Y.; Zhao, S. (2008). Simulation of spin-axis position measurement of superconducting sphere rotor by fiber optic sensor. IEEE Trans Appl Supercond., Vol 18, pp. 836-839.

Dong, X.W. \& Zhao, R.F. (2010). Detection of liquid level variation using a side polished fiber bragg grating. Optics $\mathcal{E}$ laser technology, 42, 214-218.

Elasar, J.; Selmic, S.; Tomic, M.; Prokin, M. (2002). A fiber optic displacement sensor for a cyclotron environment based on a modified triangulation method. J. Opt. A: Pure Appl. Opt., Vol. 4, pp. 347-355.

Faria, J.B. (1998). A theoretical analysis of the bifurcated fiber bundle displacement sensor. IEEE Transactions on instrumentation and measurement, Vol. 47, pp. 742-747.

Kleiza1, V. \& Verkelis, J. (2007). Some Advanced Fiber-Optic Amplitude Modulated Reflection Displacement and Refractive Index Sensors. Nonlinear Analysis: Modelling and Control, Vol. 12, No. 2, pp. 213-225.

Ko, W.H.; Chang, K.M.; Hwang, G.J. (1995). A fiber optic reflective displacement micrometer. Sensor Actuators A, Vol. 49, pp. 51-55.

Kulchin, Y.N.; Vitrik O.B.; Dyshlyuk A.V.; Shalagin, A.M.; Babin, S.A.; Vlasov, A.A. (2007). Applications of optical time domain reflectometry for the interrogation of fiber bragg sensors. Laser Physics, Vol. 17, pp. 1335-1339.

Laurence Bergougnous, Jacqueline Misguich-Ripault, and Jean-Luc Firpo, (1998). Characterization of an optical fiber bundle sensor. Rev. Sci. Instrum. 69, 1985-1990.

Lim, K.S.; Harun, S.W.; Yang, H.Z.; Dimyati, K.; Ahmad, H. (2009). Analytical and experimental studies on asymmetric bundle fiber displacement sensors. J. of Modern Optics, Vol. 56, pp.1838-1842. 
Lomer, M.; Arrue, J.; Jauregui, C.; Aiestaran, P.; Zubia, J.; Lopez-Higuera, J.M. (2007). Lateral polishing of bends in plastic optical fibers applied to a multipoint liquid level measurement sensor. Sensors and Actuators A, 137, 68-73.

Miclos, S. \& Zisu, T. (2001). Chalcogenide fibre displacement sensor. J. Optoelectron Adv. Matter., Vol. 3, pp. 373-376.

Murphy, A.M.; Gunther, M.F.; Vengsarkar, A.M.; Claus, O.R. (1991). Quadrature phaseshifted extrinsic Fabry-Perot optical fiber sensor. Opt. Lett ., 16, 273.

Murphy, M.M. \& Jones, G.R. (1994). A variable range extrinsic optical fiber displacement sensor. Pure Appl. Opt., Vol. 3, pp. 361-369.

Nalwa, S. (2004). Polymer optical fibers. California: American Scientific Publishers.

Nath, P.; Singh, H.K.; Datta, P.; Sarma, K.C. (2008). All-fiber optic sensor for measurement of liquid refractive index. Sensor and Actuators A, Vol.148, pp.16- 18.

Oiwa T. \& Nishitani, H. (2004). Three dimensional touch probe using three fiber optic displacement sensor. Meas. Sci. Technol., Vol. 15, pp. 84-90.

Rastogi, P.K. (1997). Optical Measurement Techniques and Applications. Artech House, Inc. Boston, London.

Saunders, C. \& Scully. (2007). Sensing applications for POF and hybrid fibres using a photon counting OTDR. Meas. Sci. Technol., Vol. 18, pp. 615-622.

Shimamoto, A. \& Tanaka, K. (2001). Geometrical analysis of an optical fiber bundle displacement sensor. Applied Optics, Vol. 35, Issue 34, pp. 6767-6774.

Sohn, K.R. \& Shim, J.H. (2009). Liquid level monitoring sensor system using fiber bragg grating embedded in cantilever. Sensors and Actuators, A, 152, 248-251.

Spooncer, R.C.; Butler, C.; Jones, B.E. (1992). Optical fibre displacement sensors for process and manufacturing applications. Opt. Eng. 31, 1632-1637.

Suhadolnik; Babnik, A.; Mo2ina,J. (1995). Optical fiber reflection refractometer. Sensor and Actuators B, Vol. 29, pp.428- 432.

Van Etten W. \& Van der Plaats J. (1991). Fundamentals of optical fiber communi-cations. Prentice-Hall, London.

Wang, D.X.; Karim, M.A.; Li, Y. (1997). Self referenced fiber optic sensor performance for micro displacement measurement. Opt. Eng., Vol. 36, pp. 838-842.

Yang, H.Z.; Lim, K.S.; Harun, S.W.; Dimyati, K.; Ahmad, H. (2010). Enhanced Bundle Fiber Displacement Sensor Based on Concave mirror. Sensors and Actuators A, 162, 8-12.

Yang, H.Z.; Harun, S.W.; Ahmad, H. (2009). Fiber Optic Displacement and Liquid Refractive Index Sensors with Two Asymmetrical Inclined Fibers. Sensor and Transducer, Vol.108, pp. 80-88.

Yasin,M.; Harun, S.W.; Pujiyanto, Ghani, Z.A.; Ahmad H. (2010). PerformanceComparison between Plastic-Based Fiber Bundle and Multimode FusedCoupler as Probes in Displacement Sensors. Laser Physics, Vol. 20, No. 10, pp. 1890-1893.

Yasin, M.; Harun, S.W.; Abdul-Rashid, H.A.; Kusminarto; Karyono; Ahmad, H. (2008). The performance of a fiber optic displacement sensor for different types of probes and targets. Laser Physics Letters, Vol. 5, No. 1, pp. 55-58.

Yasin, M.; Harun, S.W.; Samian; Kusminarto; Ahmad, H. (2009). Simple design of optical fiber displacement sensor using a multimode fiber coupler. Laser Physics, Vol. 19, No.7, pp. 1446-1449.

Yun, B.F.; Chen, N.; Cui, Y.P. (2007). Highly sensitive liquid level sensor based on etched fiber bragg grating. IEEE Photonic Technology Letters, 19, 1747-1749. 


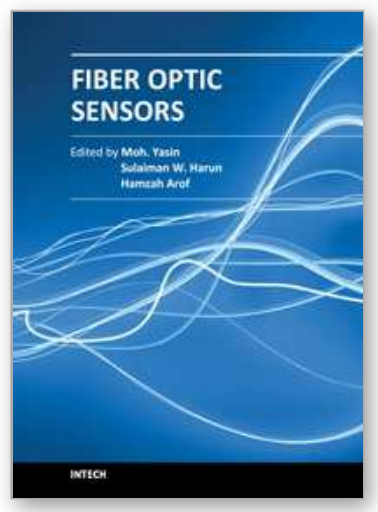

\author{
Fiber Optic Sensors \\ Edited by Dr Moh. Yasin
}

ISBN 978-953-307-922-6

Hard cover, 518 pages

Publisher InTech

Published online 22, February, 2012

Published in print edition February, 2012

This book presents a comprehensive account of recent advances and researches in fiber optic sensor technology. It consists of 21 chapters encompassing the recent progress in the subject, basic principles of various sensor types, their applications in structural health monitoring and the measurement of various physical, chemical and biological parameters. It also highlights the development of fiber optic sensors, their applications by providing various new methods for sensing and systems, and describing recent developments in fiber Bragg grating, tapered optical fiber, polymer optical fiber, long period fiber grating, reflectometry and interefometry based sensors. Edited by three scientists with a wide knowledge of the field and the community, the book brings together leading academics and practitioners in a comprehensive and incisive treatment of the subject. This is an essential reference for researchers working and teaching in optical fiber sensor technology, and for industrial users who need to be aware of current developments and new areas in optical fiber sensor devices.

\title{
How to reference
}

In order to correctly reference this scholarly work, feel free to copy and paste the following:

S. W. Harun, M. Yasin, H. Z. Yang and H. Ahmad (2012). Fiber Optic Displacement Sensors and Their Applications, Fiber Optic Sensors, Dr Moh. Yasin (Ed.), ISBN: 978-953-307-922-6, InTech, Available from: http://www.intechopen.com/books/fiber-optic-sensors/fiber-optic-displacement-sensors-and-their-applications

\section{INTECH}

open science | open minds

\section{InTech Europe}

University Campus STeP Ri

Slavka Krautzeka 83/A

51000 Rijeka, Croatia

Phone: +385 (51) 770447

Fax: +385 (51) 686166

www.intechopen.com

\section{InTech China}

Unit 405, Office Block, Hotel Equatorial Shanghai

No.65, Yan An Road (West), Shanghai, 200040, China

中国上海市延安西路65号上海国际贵都大饭店办公楼 405 单元

Phone: +86-21-62489820

Fax: $+86-21-62489821$ 
(C) 2012 The Author(s). Licensee IntechOpen. This is an open access article distributed under the terms of the Creative Commons Attribution 3.0 License, which permits unrestricted use, distribution, and reproduction in any medium, provided the original work is properly cited. 